\title{
Progression to AIDS in SIV-infected rhesus macaques is associated with distinct $K I R$ and $M H C$ class I polymorphisms and NK cell dysfunction
}

\author{
Christina Albrecht ${ }^{1+}$, Dörthe Malzahn ${ }^{2+}$, Markus Brameier ${ }^{1}$, Meike Hermes $^{1}$, Aftab A. Ansari ${ }^{3+}$ and \\ Lutz Walter ${ }^{1+*}$
}

1 Primate Genetics Laboratory, German Primate Center, Leibniz-Institute for Primate Research, Göttingen, Germany

2 Department of Genetic Epidemiology, University Medical Center, Georg-August-University, Göttingen, Germany

${ }^{3}$ Department of Pathology and Laboratory Medicine, Emory University School of Medicine, Atlanta, GA, USA

\section{Edited by:}

Carsten Watzl, Leibniz Research

Centre for Working Environment and

Human Factors - IfADo, Germany

Reviewed by:

Subramaniam Malarkannan, Medical

College of Wisconsin, USA

Salim Iqbal Khakoo, University of

Southampton, UK

Marcus Altfeld, Ragon Institute of

MGH, MIT and Harvard, USA

\section{*Correspondence:}

Lutz Walter, German Primate Center, Leibniz-Institute for Primate Research, Kellnerweg 4, Göttingen 37077,

Germany

e-mail:walter@dpz.eu

${ }^{+}$Christina Albrecht and Dörthe Malzahn share first authorship; Aftab A. Ansari and Lutz Walter share senior authorship.
Killer cell immunoglobulin-like receptors (KIR) regulate the activity of natural killer (NK) cells and have been shown to be associated with susceptibility to a number of human infectious diseases. Here, we analyzed NK cell function and genetic associations in a cohort of 52 rhesus macaques experimentally infected with SIVmac and subsequently stratified into high viral load (HVL) and low viral load (LVL) plasma viral loads at set point. This stratification coincided with fast (HVL) and slow (LVL) disease progression indicated by the disease course and critical clinical parameters including CD4+T cell counts. HVL animals revealed sustained proliferation of NK cells but distinct loss of peripheral blood NK cell numbers and lytic function. Genetic analyses revealed that KIR genes 3DL05, 3DSO5, and $3 D L 10$ as well as 3DSW08, 3DLW03, and 3DSW09 are correlated, most likely due to underlying haplotypes. SIV-infection outcome associated with presence of transcripts for two inhibitory KIR genes (KIR3DLO2, KIR3DL 10) and three activating KIR genes (KIR3DSW08, KIR3DSO2, KIR3DSO5). Presence of KIR3DLO2 and KIR3DSW08 was associated with LVL outcome, whereas presence of KIR3DSO2 was associated with HVL outcome. Furthermore, we identified epistasis between KIR and MHC class I alleles as the transcript presence of the correlated genes KIR3DL05, KIR3DS05, and KIR3DL 10 increased HVL risk when Mamu- $B^{*} 012$ transcripts were also present or when Mamu-A1*001 transcripts were absent. These genetic associations were mirrored by changes in the numbers, the level of proliferation, and lytic capabilities of NK cells as well as overall survival time and gastro-intestinal tissue viral load.

Keywords: virus infection, killer cell immunoglobulin-like receptors, MHC class I ligands, genetic polymorphism, epistasis, rhesus macaque SIV infection model, AIDS

\section{INTRODUCTION}

Natural killer (NK) cells are large bone marrow-derived granular lymphocytes that perform essential functions in the innate immune response against various pathogens and in the priming of adaptive immunity. The main effector functions of NK cells are target cell killing and cytokine release, which are regulated via integration of signals derived from stimulatory and inhibitory receptors expressed on the cell surface of these lymphocytes. NK cells are important effector cells to combat infection with viruses (1), including human and simian immunodeficiency viruses (SIVs) (2-4).

Killer cell immunoglobulin-like receptors (KIR) are crucial regulators of NK cell activity and exhibit considerable allelic polymorphism and copy-number variations in humans and higher primates $(5,6)$. Epidemiological studies of human immunodeficiency virus 1 (HIV-1) infection indicate that a combination of HLA-Bw4-80I with either KIR3DL1 or KIR3DS1 is associated with delayed progression to AIDS (3, 7-9). Differences in expression levels of KIR3DL1 allotypes correlate with different functional capacities of NK cells (10), and KIR3DL1 allotypes expressed at high levels are associated with delayed progression to AIDS (11). While the role of inhibitory KIR3DL1 in the regulation of NK cell activity toward HIV-1-infected cells is plausible, the molecular basis underlying the epistasis of KIR3DS1 with HLA-Bw4-80I is unknown and any demonstration of physical interaction between KIR3DS1 and HLA-Bw4 proteins has so far been unsuccessful. Nevertheless, KIR3DS1-positive NK cells were reported to inhibit HIV-1 replication more effectively on target cells expressing HLA-Bw4-80I compared to KIR3DS1-negative NK cells (12). In addition, both KIR3DS1- and KIR3DL1-positive NK cell proportions are elevated during acute HIV-1 infection in the presence of HLA-Bw4-80I molecules (13). A recent paper by Song and colleagues (9) confirmed these findings and demonstrated the impact of the strength of educational signals obtained via the KIR3DL1 receptor and an important role of CC-chemokine release to inhibit HIV-1 replication. Further support for the impact of NK cells on HIV-1 infection was documented in studies of HIV1 peptide escape mutations that resulted in stronger interaction 
of the inhibitory KIR2DL2 with HLA-C1 presenting mutated peptides (14).

Simian immunodeficiency virus (SIV)-infected rhesus macaques represent an established and important animal model to study the mechanisms of HIV infection. SIV-induced syndromes and the temporally diverse progression to AIDS of SIV-infected rhesus macaques are remarkably similar to human HIV-1 infection with variable disease outcome ranging from spontaneous control of plasma viremia (elite controllers, about $5-20 \%$ of infected individuals) to uncontrolled viremia and rapid disease course (fast progressors, about 5-10\%). These polarized clinical outcomes during persistent viremia in experimentally infected rhesus macaques suggest genetic variability may play a critical role in SIV containment. Rhesus macaque KIR genes are considered as candidate genes that contribute to different clinical outcomes as they are at least as diverse as human KIR genes (15-18). Indeed, in SIVinfected rhesus macaques, copy number variations (CNV) of the inhibitory KIR3DL05 gene were shown to be associated with fast progression to AIDS, whereas CNVs of activating KIR3DS genes were associated with control of virus replication $(19,20)$. However, it is clear that the studies of the beneficial or detrimental combinations of KIR and MHC class I polymorphisms are in their infancy and much has yet to be learnt about these associations. Further, it is unclear whether changes in the level of expression of KIRs occur during the course of SIV infection and whether these changes contribute to disease pathogenesis.

Herein, we demonstrate that NK cells in SIV-infected rhesus macaques with rapid disease progression and high viral load (HVL) show sustained proliferation but less lytic activity, followed by a dramatic depletion of NK cells from the peripheral blood. This functional impairment is accompanied by changes of transcription of five KIR genes (inhibitory: KIR3DL02, KIR3DL10; activating: KIR3DSW08, KIR3DS02, KIR3DS05). Presence of the correlated KIR3DL05, KIR3DSO5, and KIR3DL10, genes combined with presence of $M a m u-B^{\star} 012$ or with lack of $M a m u-A 1^{\star} 001$ was more frequently found in animals rapidly progressing to disease, and animals with such KIR/MHC class I combinations displayed higher gastro-intestinal tissue (GIT) viral loads and shorter survival times.

\section{MATERIALS AND METHODS ETHICAL STATEMENT}

Juvenile to adult male rhesus macaques (Macaca mulatta) of Indian origin were used for the studies reported herein. All animals were born and maintained at the Yerkes National Primate Research Center of Emory University (Atlanta, GA, USA) in accordance with the regulations of the Committee on the Care and Use of Laboratory Animal Resources, National Research Council and the Department of Health and Human Service guideline titled Guide for the Care and Use of Laboratory Animals, and with the Weatherall report (21). None of the animals, blood and tissue samples originated from the German Primate Center. The animals were fed monkey diet (Purina) supplemented daily with fresh fruit or vegetables and water ad libitum. Additional enrichment including the delivery of appropriate safe toys is provided and overseen by the Yerkes enrichment staff and animal health is monitored daily by the animal care staff and veterinary personnel, available 24/7. Monkeys are caged in socially compatible same sex pairs to facilitate social enhancement and well-being. Monkeys showing signs of sustained weight loss, disease or distress are subject to clinical diagnosis based on symptoms and then provided either standard dietary supplementation analgesics and/or chemotherapy. Monkeys whose symptoms cannot be alleviated using standard dietary supplementation, analgesics and/or chemotherapy were humanely euthanized using an overdose of barbiturates according to the guidelines of the American Veterinary Medical Association. The studies reported herein were performed under IACUC protocol \#2001186 "Innate immunity in SIV infection" which was reviewed and approved by the Emory University IACUC. It has been assigned the IACUC protocol number "YER-2001186-082414GA." The Yerkes National Primate Research Center has been fully accredited by the Association for Assessment and Accreditation of Laboratory Animal Care International since 1985. All experiments were reviewed and approved by the Emory institutional animal use and care as well as biosafety review Committees.

\section{SIV INFECTION OF RHESUS MACAQUES}

This study was conducted on a cohort of 52 juvenile to adult male rhesus macaques that were infected intravenously with 1000 TCID50 of either SIVmac251 $(n=5)$ or SIVmac239 $(n=47)$ grown in con-A blast cultures from normal rhesus macaques. Statistical analyses in this report results were fairly robust when excluding the five SIVmac251 infected animals (data not shown). On the other hand including these animals notably increased statistical power by increasing the sample size. Based on viral loads following achievement of stable set points (that correlated with absolute peripheral blood CD4+ T cell levels, and clinical characteristics including hemogram values, weight gain/loss and blood chemistries, see Table 1) the cohort was stratified into rhesus macaques with low plasma viral load (LVL $(n=24) ;<10^{5}$ mean \pm S.D. viral copies/mL of plasma; slow progression to AIDS) and rhesus macaques with high plasma viral load (HVL $(n=28)$; $>10^{5}$ mean \pm SD viral copies $/ \mathrm{mL}$ of plasma; rapid progression to AIDS). Figure 1 shows box and whiskers plots of viral loads at set point, indicating that the virus copy numbers are significantly different $(p=0.0008)$ and non-overlapping between the two strata, and Figure 2 underlines the distinctly different survival times between HVL and LVL. Blood samples from each monkey were obtained three times prior to SIV infection to derive average baseline absolute numbers of the various cell lineages. The monkeys were bled once per week post infection for 6-8 weeks and then biweekly for an additional 2 months and monthly thereafter to monitor plasma viral copy numbers and absolute numbers of lymphoid cell subsets. Cellular viral loads in aliquots of PBMC and colorectal biopsy tissue samples were monitored using a technique standardized in our lab that included negative and positive controls.

\section{SPECIMEN COLLECTION, MONONUCLEAR CELLS ISOLATION, VIRUS QUANTITATION}

Peripheral blood mononuclear cells (PBMC) were isolated from heparinized whole blood using standard Ficoll-Hypaque centrifugation procedure. The cell count and cell viability were 
Table 1 | Overview of the macaque cohort $(n=52)$

\begin{tabular}{|c|c|c|c|c|c|c|c|}
\hline \multirow{2}{*}{$\begin{array}{l}\text { Outcome } \\
\text { Virus }^{a}\end{array}$} & \multicolumn{3}{|c|}{ LVL, $n=24$} & \multicolumn{4}{|c|}{ HVL, $n=28$} \\
\hline & SIVmac239, $n=22$ & \multicolumn{2}{|c|}{ SIVmac251, $n=2$} & SIVmac239, $n=25$ & \multicolumn{3}{|c|}{ SIVmac251, $n=3$} \\
\hline & Median (range) & $\mathrm{mm} 34$ & mm38 & Median (range) & $\mathrm{mm} 19$ & $\mathrm{~mm} 25$ & $\mathrm{~mm} 27$ \\
\hline Sex & $100 \%$ male & Male & Male & $100 \%$ male & Male & Male & Male \\
\hline & 1492 (median) & 1680 & 2061 & 1555 (median) & 1695 & 1810 & 1801 \\
\hline & $658-4549$ (min-max) & & & 1024-4570 (min-max) & & & \\
\hline \multicolumn{8}{|c|}{ Intravenously injected viral dose: 1000 TCID50 per animal } \\
\hline \multicolumn{8}{|c|}{$C D 4^{+} T$ cell number in peripheral blood } \\
\hline \multicolumn{8}{|c|}{ Viral load in peripheral blood } \\
\hline At peak & $2.2 \times 10^{6}\left(4.2 \times 10^{3}-32.6 \times 10^{6}\right)$ & $0.3 \times 10^{6}$ & $1.1 \times 10^{6}$ & $15.0 \times 10^{6}\left(2.8 \times 10^{6}-104.0 \times 10^{6}\right)$ & $10.8 \times 10^{6}$ & $21.0 \times 10^{6}$ & $6.7 \times 10^{6}$ \\
\hline At set point & $2.4 \times 10^{3}\left(20-70.2 \times 10^{3}\right)$ & $4.0 \times 10^{3}$ & $1.5 \times 10^{3}$ & $3.3 \times 10^{6}\left(0.3 \times 10^{6}-36.8 \times 10^{6}\right)$ & $3.0 \times 10^{6}$ & $4.0 \times 10^{6}$ & $2.0 \times 10^{6}$ \\
\hline
\end{tabular}

Outcomes of interest

Survival time (in month, censored times indicated by $>$ )

\begin{tabular}{|c|c|c|c|c|c|c|c|}
\hline Median & $>200$ month & $>73$ & $>156$ & 38 month & 151 & $>14$ & 54 \\
\hline Last event & - & & & 70 month & & & \\
\hline \# Events & 1 event & & & 26 events & & & \\
\hline \multicolumn{8}{|c|}{ Viral load in gastro-intestinal tissue } \\
\hline \multicolumn{8}{|c|}{ Number of NK cells } \\
\hline At pre-infection & $860.5(668-1229)$ & 831 & 729 & $884.0(612-1242)$ & 933 & 893 & 789 \\
\hline$\geq 26$ weeks p.i. & $639.5(348-834)$ & 332 & 673 & $94.0(32-143)$ & 87 & 124 & 143 \\
\hline \multicolumn{8}{|l|}{$\% K i 67+N K$ cells } \\
\hline \multicolumn{8}{|c|}{ \% CD107a-positive NK cells } \\
\hline Pre-infection & $18.3(4.9-37.2)$ & Missing & 22.5 & $23.7(6.9-42.8)$ & 32.7 & 13.9 & 19.3 \\
\hline Acute infection & $18.6(12.3-27.6)$ & Missing & 15.4 & $16.8(7.2-33.9)$ & 13.2 & 14.3 & 6.9 \\
\hline Chronic infection & $16.2(8.1-30.1)$ & Missing & 16.9 & $8.0(0.1-24.6)$ & 6.9 & 2.1 & 0.4 \\
\hline
\end{tabular}

While aminals were comparable at pre-infection, the cohort separated into two distinct subgroups (LVL, HVL) regarding set point viral loads. All other clinical parameters consistently indicate slow (LVL) or fast (HVL) disease progression.

${ }^{a}$ Virus strain did not associate with infection outcome (HVL outcome: $p=1.00$, survival time: $p=0.591$ ).

performed using the trypan blue dye technique. The cell concentrations were adjusted to $1 \times 10^{6}$ cells $/ \mathrm{mL}$ in complete RPMI1640 media. Aliquots of EDTA plasma were obtained prior to and at varying intervals following SIV infection and sent on dry ice to Siemens Laboratories (Siemens, Berkeley, CA, USA) for quantitating plasma SIV levels using the branched DNA assay. Data 

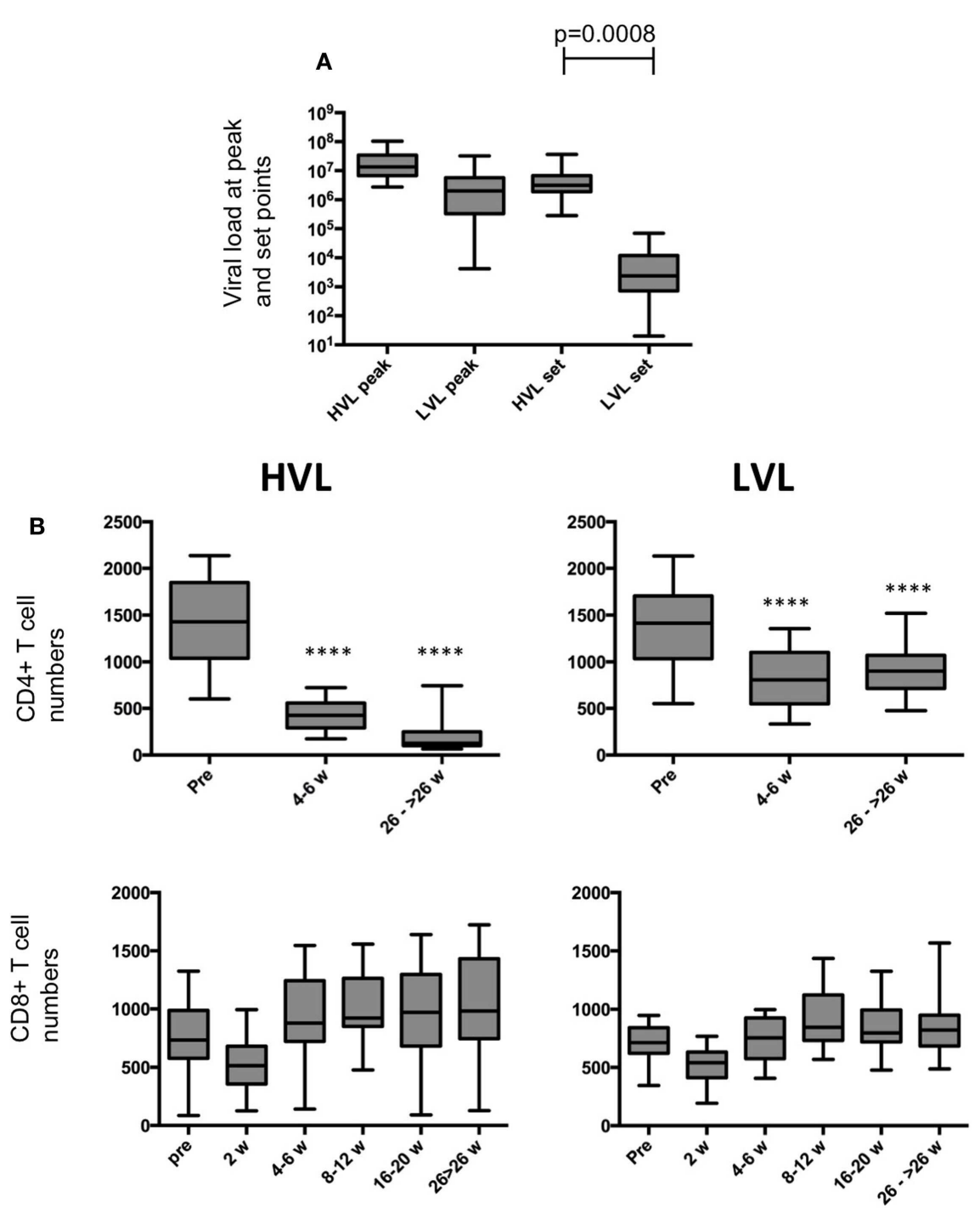

FIGURE 1 | Viral loads and T cell numbers. (A) Plasma viral loads at peak and set point in HVL and LVL animals. Viral loads of HVL and LVL animals differ significantly at set point ( $t$-test, $p=0.0008$ ). (B) Box and whisker plots of CD4+
$T$ cell and CD8+T cell numbers during course of SIV infection in peripheral blood of HVL and LVL animals. T-tests were performed between the cell numbers of corresponding time points and were indicated as ${ }^{* * *} p<0.0001$. obtained are illustrated as plasma VL per milliliter of plasma. For the quantitation of pro-viral DNA load, aliquots of the unfractionated PBMCs and cells obtained from the colorectal gut biopsy tissues collected at various times post infection were used to isolate total genomic DNA using the Qiagen DNAeasy kit (Qiagen, Valencia, CA, USA). For purposes of brevity, only the data for pro-viral DNA levels on cells isolated from rectal biopsy samples obtained during viral load set point (week 8 p.i.) are shown. An aliquot of the genomic DNA isolated was used to quantitate the amount of DNA per sample, and the number of viral copies per nanogram of DNA was determined using our standardized laboratory protocol for quantitation of SIV gag $(22,23)$. The SIV1C cell line that has a single copy of SIV DNA per cell was used as a control for the pro-viral DNA analysis. The sensitivity of the assay was one infected cell per million. The quantitation of viral RNA was performed using RNA extraction, RNAse free primer pairs, and real-time PCR utilizing SYBR Green for iCycler kit as previously described (24). The sensitivity of this assay was 10 viral copies/mL.

\section{POLYCHROMATIC FLOW CYTOMETRY AND} FLUOROCHROME-CONJUGATED MONOCLONAL ANTIBODIES

Various fluorochrome-conjugated monoclonal antibodies (mAbs) were used in appropriate combinations to stain aliquots of the PBMCs. The mAbs utilized were purchased from BD Biosciences (San Jose, CA, USA) and included Alexa700-anti-CD3 (clone SP34-2), PerCP, Pac Blue or V450-conjugated anti-CD4 (clone L200), FITC or V500-anti-CD8 (clones SK1, RPA-T8), FITCanti-CD14 (clone M5E2), PerCP-Cy5.5 or APC-Cy7-anti-CD16 (clone 3G8), APC-Cy7 or PeE-Cy7-anti-CD20 (clones 2H7, L27), PE-Cy7-anti-CD56 (clone NCAM16.2), APC-anti-CD123 (clone 

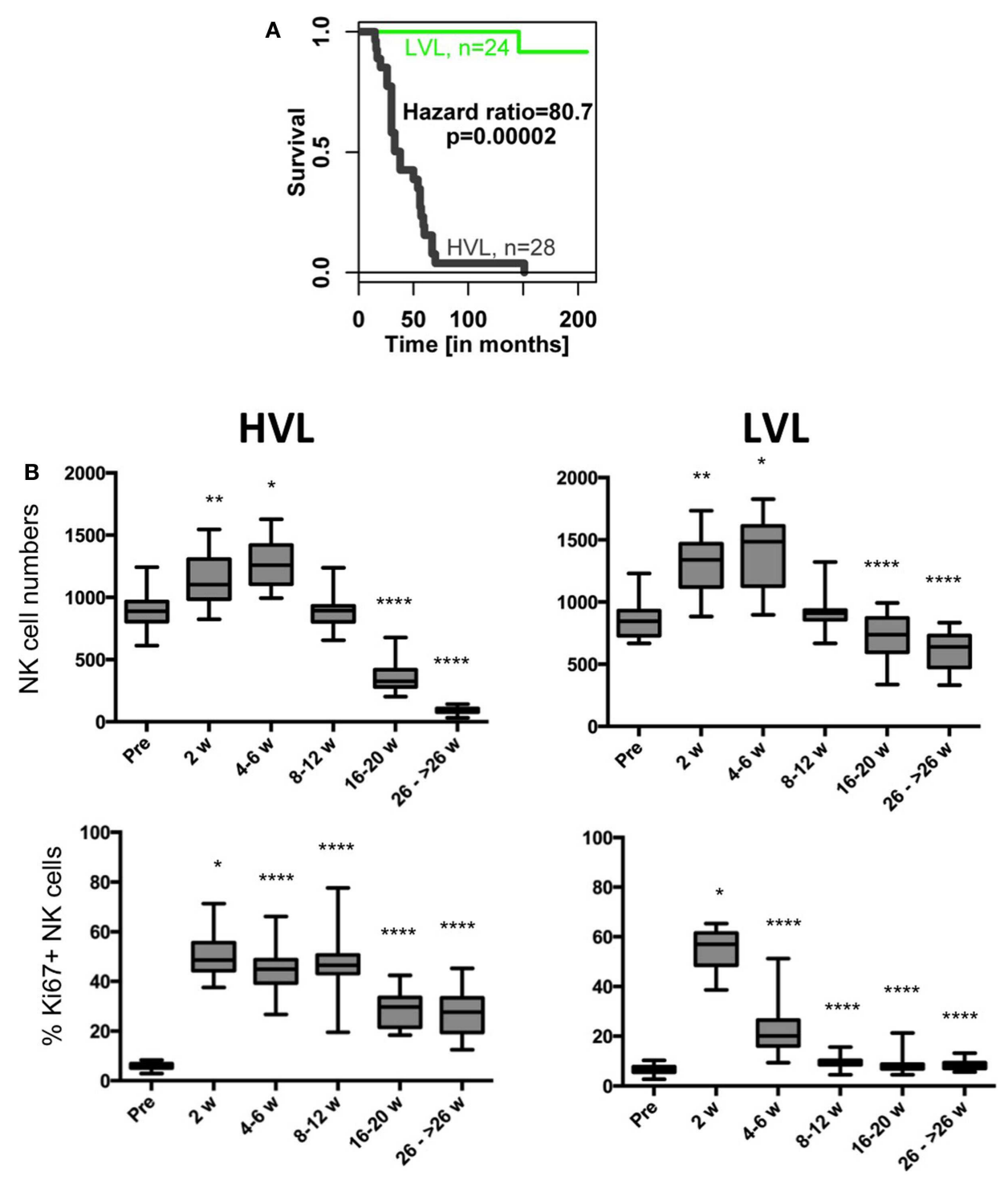

FIGURE 2 | Survival times as well as NK cell counts and percentage of Ki67-positive NK cells during the course of SIV infection in HVL and LVL animals. (A) Kaplan-Meier survival curve of both strata. (B) Box and whisker plots of NK cell numbers (upper panel) and \% Ki67-positive NK cells (lower panel). Sampling of cells was before infection (pre) and at indicated weeks after infection. $t$-Tests were performed between the cell numbers of corresponding time points and were indicated as ${ }^{*} p<0.05 ;{ }^{* *} p<0.01$; **** $p<0.0001$.
7G3), PerCP-Cy5.5-anti-HLA-DR (clone L243), FITC or PEconjugated Ki67 (clone B56), Alexa700 or PE-Cy5-anti-CD107a (clone H4A3). The PE-anti-NKG2A (clone Z199) was purchased from Beckman Coulter (Brea, CA, USA) and FITC-anti-human $\gamma \delta$ TCR (clone SA6.E9) from Invitrogen. Standard gating strategies were utilized to identify CD3 $+\mathrm{CD} 4+\alpha \beta \mathrm{T}$ cells, $\mathrm{CD} 3+\mathrm{CD} 8+$ $\alpha \beta T$ cells, CD3 $+\gamma \delta$ T cells, CD3 - CD20 + B cells, CD3 - CD8$\mathrm{NKG} 2 \mathrm{~A}-\mathrm{CD} 14+$ monocytes, as well as CD3-CD8+ NKG2A+ NK cells and respective subsets based on CD16 and CD56 expression $(25,26)$. The cells were incubated with the appropriate combination of fluorochrome-conjugated antibodies for $30 \mathrm{~min}$ covered with aluminum foil and kept at $4^{\circ} \mathrm{C}$. The cells were then washed twice with FACS buffer and resuspended in FACS buffer containing 2\% paraformaldehyde and kept under aluminum foil until analyzed using a LSRII flow cytometer (BD Immunocytometry Division, Mountain View, CA, USA). A minimum of 100,000 events were examined per sample and data were analyzed using FlowJo software (TreeStar, Ashland, OR, USA). An aliquot of the blood sample was utilized to perform complete blood count and the values utilized to calculate absolute number of each cell lineage.

\section{NK CELL FUNCTION ANALYSIS}

An aliquot of the PBMC sample to be analyzed for NK functional activity was co-cultured in vitro with HLA class I-devoid $\mathrm{K} 562$ or 721.221 cells at an effector/target ratio of $10: 1$ for $6 \mathrm{~h}$ at $37^{\circ} \mathrm{C}$ in a humidified $7 \%$ CO2 incubator in the presence of Brefeldin-A 
(5 ug/mL, Sigma-Aldrich), Monensin (6 ug/mL, Golgi-Stop, BD Biosciences) and anti-CD107a (clone LAMP1, BD Biosciences). Media throughout consisted of RPMI 1640 supplemented with $10 \%$ fetal calf serum, $2 \mathrm{mM}$ L-glutamine, 100 units $/ \mathrm{mL}$ penicillin and $100 \mathrm{ug} / \mathrm{mL}$ streptomycin. Each assay included an aliquot of the same PBMC cultured alone (negative control) and an aliquot of the same PBMC incubated with $1.25 \mathrm{ug} / \mathrm{mL}$ phorbol-12-myristate 13acetate (PMA, Sigma-Aldrich) and $0.25 \mathrm{ug} / \mathrm{mL}$ ionomycin (Sigma Aldrich) as a positive control. Only experiments in which the negative control gave $<10 \%$ value and $>3$-fold increase between the negative and positive control were considered valid. After the co-culture, the cells were stained for viability using the Aqua LIVE/DEAD (Invitrogen) and stained with anti-CD3, CD8 alpha, NKG2A, CD16 and CD56 antibodies. The cells were washed and resuspended in the FACS buffer and analyzed using the LSRII flow cytometer. A minimum of 100,000 events were analyzed and the frequency of the gated population of CD3-, CD8+, NKG2A+ CD16+ cells that were CD107a+ determined using FlowJo software (TreeStar, Ashland, OR, USA). Data obtained in the experimental sample - the negative control was calculated and utilized to illustrate NK cell functional activity.

\section{NEXT-GENERATION SEQUENCING OF MHC CLASS I AND KIR GENE TRANSCRIPTS}

MHC class $I$ and KIR gene transcripts were analyzed from PBMC cDNA samples using Roche/454 Titanium chemistry in a Roche/454 GS Junior sequencer (Roche Applied Science) according to previously published methods $(18,27)$. Amplicon library preparation, emPCR, bead recovery and sequencing were performed according to the manufacturer's instructions. Pools of twelve samples were sequenced on a single PicoTiterPlate. The emPCR conditions for MHC class I and for KIR amplicon libraries were performed following the standard protocol instructions and recommendations for sequencing of long-length amplicon libraries ( $\geq 550 \mathrm{bp}$ ), respectively. The ratio of DNA and capture beads used for emPCR was in the order of 1-2 molecules per bead.

For each individual, separate library files were generated by sorting of sequencing reads with identical multiplex identifiers (MID). Primer sequences were cut off. Only reads with identical sequences that occurred at least five times were considered in order to reduce sequencing errors. The filtered reads were aligned against reference sequences of all known rhesus macaque $\mathrm{MHC}$ class I alleles (IPD MHC database non-human primates http: //www.ebi.ac.uk/ipd/mhc/nhp/index.html) and rhesus macaque KIR database (kindly obtained from Dr. Libby Guethlein, Stanford University, USA) using BLAST. Perfectly matching reads were identified from MHC class I and KIR gene databases. In order to detect alleles that are not included in these databases, a maximum of two mismatches compared to the reference sequences were allowed. As forward and reverse reads were aligned separately, sequences with a maximum of four mismatches, over the full read length were included in the final preparation of KIR transcription profiles of each rhesus macaque. Since the region of the sequenced KIR transcripts of about $560 \mathrm{nt}$ (623 nt with primer regions) is not fully covered by a single read, sufficient counts $(\geq 5)$ of matching forward and reverse reads needed to be found.
The overlap between forward and reverse reads covers around one third (180 nt) of the sequenced part of the KIR gene transcript. We also tested the KIR profile allowing four or six mismatches (i.e., 8 or 12 in forward + reverse), but this lead to ambiguous alignments for some KIR gene transcripts, i.e., matching of single reads to multiple KIR genes (data not shown). Frequencies of filtered forward and reverse reads were summed up and the relative contribution of each KIR transcript was compared to the total number of KIR transcripts identified in each individual. The MHC class $I$ and $K I R$ gene transcript sequencing data of the entire 52 rhesus macaques are shown in Tables S1 and S2 in Supplementary Material.

\section{STATISTICAL ANALYSES}

The various statistical software programs used included SAS, R, and GraphPad Prism 6.

Time-course of NK cell numbers from 8-12 weeks p.i. to 26 to $>26$ weeks p.i. was analyzed by longitudinal linear regression, testing statistical interaction between covariates time and group (HVL, LVL) (see Figure 2). Difference of means of \% Ki67 + NK cells at 8-12 weeks p.i. between HVL and LVL animals was examined by t-test; and the lytic function of NK cells was analyzed in HVL and LVL by repeated measures ANOVA (Figure 3, testing change of mean\%CD107a-positive NK cells over time, i.e., from pre- to acute and chronic infection).

Target HVL outcome was analyzed by logistic regression, survival times by Cox regression, and NK cell number and corresponding functional data as well as viral loads in gut tissue by linear regression models, testing KIR main effects (Table 3, multiple or single $K I R$ covariates as indicated) and $K I R-M H C$ class $I$ epistasis (see Table 4 and Figures 5 and 6; statistical interaction models contained a KIR and a MHC-I covariate, respectively). Furthermore, for the main target HVL outcome, we compared a multiple model (multi-KIR) with univariate analyses (single-KIR) (see Table 3,

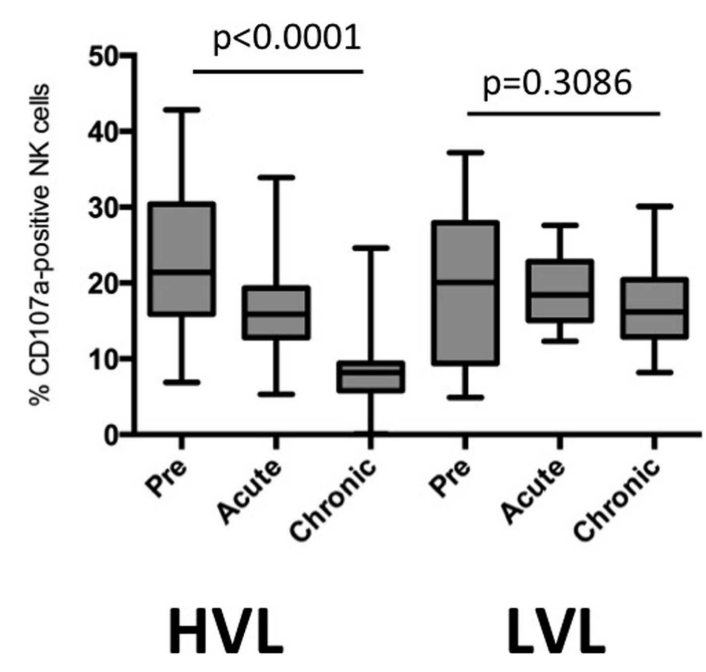

FIGURE 3 | Cytotoxic capacity of NK cells during different phases of SIV infection in HVL and LVL animals. Degranulation after stimulation with $\mathrm{MHC}$ class I-deficient cells was determined as \% CD107a-positive NK cells. Acute and chronic phases represent time points 2-4 weeks and $>16$ weeks after infection. 
first columns). In contrast to single-KIR analyses, the multi-KIR model estimates mutually adjusted $K I R$ main effects and accounts for correlations between KIR genes ( $a$ priori excluded were KIR genes that had small strata size or strong correlations with other $K I R$ genes). In addition, the frequency of KIR transcript sequence reads at pre- and acute-infection (where NK cell numbers were high and comparable across HVL and LVL) was jointly analyzed in KIR-positive animals as longitudinal metric target by longitudinal rank-sum test (28) for association with HVL outcome (Table 3, last column). Rank-sum testing ensured test validity given that KIR transcript frequencies had skewed distributions. The rank-sum test resembles a heteroscedastic 1-way repeated measures ANOVA with factor HVL outcome on the longitudinal rank-order data of observed KIR transcript frequencies. Longitudinal analyses incorporated all animals that had any trait data (number of animals is indicated in Table 3; no omission of animals with incomplete follow-up and no imputation of missing values). We used SAS procedure mixed for longitudinal linear regression on\% CD107apositive NK cells and R package nparLD for longitudinal rank-sum testing of relative KIR transcript amounts (Table 3 ).

Association between MHC class I alleles and HVL outcome was tested by Fisher's exact tests (see Table S3 in Supplementary Material). Finally, the probability to obtain $\mathrm{K} p$ values $\leq 0.05$ for a family of $\mathrm{M}$ association tests with HVL outcome was estimated under the null hypothesis of no associations, based on 10,000 data replicates with randomly permuted HVL/LVL group assignment.

\section{RESULTS \\ NK CELL NUMBERS, PROLIFERATION, AND ACTIVITY DURING SIV INFECTION}

The cohort of rhesus macaques $(n=52)$ was experimentally infected with SIV (SIVmac) and subsequently stratified into animals with "low viral load" (LVL) and "high viral load" (HVL) based on viral load at set point (Figure 1A). Disease progressed much faster in HVL compared to LVL as indicated by absolute levels of blood CD4+ T cell counts and other clinical parameters (see Table 1 and Materials and Methods for details). The marked differences between HVL and LVL animals are further exemplified by a Kaplan-Meier survival curve (hazard ratio 80.7, $p=0.00002$; Figure 2A). Table 1 shows a summary of the cohort and relevant data that are also displayed in Figures 1-3.

The disease course in HVL and LVL animals displays the typical marked $(p<0.0001)$ decline of CD4 + T cell counts in both strata (Figure 1B). Whereas the numbers of CD8 $+\mathrm{T}$ cells were rather similar (Figure 1B), we observed differences in blood NK cell numbers in LVL and HVL animals (Figure 2B). An increase of NK cell numbers during the acute phase of SIV infection (2-6 weeks p.i.) was followed by a steady decline in both strata. However, in contrast to LVL animals, HVL animals displayed a more marked decline in the number of NK cells during the chronic phase of infection by -30.6 per week (95\% CI $[-36.3,-24.9]$; $p<0.0001)$. At 26 weeks p.i. and beyond, the mean absolute number of NK cells per $\mu \mathrm{L}$ blood in the HVL stratum was lower by -547.2 (95\% CI $[-608.6,-485.9] ; p<0.0001)$ compared to LVL animals. The proliferation of NK cells $(\% \mathrm{Ki} 67+)$ peaked during the acute phase of infection (2 weeks p.i.) in both strata (Figure 2B). Interestingly, the percentage of Ki67 + NK cells rapidly declined in LVL animals and reached pre-infection levels at $8-12$ weeks p.i. with a mean value of $9.7 \%$ (95\% CI $[8.7,10.8])$, whereas in contrast a significantly higher amount of proliferating NK cells was observed in HVL animals as long as $8-12$ weeks p.i. ( $t$-test, $p<0.0001$ ) with a mean value of $46.4 \%(95 \%$ CI $[42.3,50.4])$ Ki67+ NK cells that was sustained significantly $(p<0.0001)$ at high levels during the chronic phase of infection. Thus, blood NK cells in HVL animals had higher proliferation activity than in LVL animals particularly at time points $4-12$ weeks p.i., a time interval when NK cell numbers of both strata are still comparable.

Next we determined the capacity of NK cells from LVL and HVL animals to lyse MHC class I-deficient cells in a degranulation assay using samples obtained at different time points post infection. The percentage of CD107a + NK cells during transition from pre-infection to acute and chronic phases declined in HVL animals (repeated measures ANOVA, $p<0.0001$ ), but remained stable in LVL animals ( $p=0.3086)$ (Figure 3).

Collectively, these data demonstrate that NK cells from animals with fast progression to AIDS and shorter survival times had sustained proliferation after SIV infection, but lost their lytic capability and constantly decreased in number following peak viral load.

\section{KIR HETEROGENEITY IN THE RHESUS MACAOUE COHORT}

As KIR proteins essentially regulate the activity of NK cells, we determined the KIR gene transcripts by next-generation sequencing (Table S1 in Supplementary Material). Baseline values obtained pre-infection, showed similar NK cell numbers that ensured comparable transcript detection sensitivity across all animals. We found a similar gene diversity and frequency distribution of transcript-positive animals in our cohort as previously published by others $(17,18)$ for Indian rhesus macaques (Figure $4 A$ ). The most frequently transcribed KIR gene in our cohort was KIR3DL01 (92\%, $n=48)$, KIR genes particularly rarely transcribed were KIR3DL04 (2\%, $n=1)$, KIR3DL06 (4\%, $n=2)$, KIR3DSO1 (12\%, $n=6)$, KIR3DSO4 (10\%, $n=5)$, and KIR3DSO6 (13\%, $n=7)$. Similar to the cohort analyzed by Moreland and co-workers (18), we detected no transcripts of the KIR3DSO7 gene in our cohort. Thus, the KIR gene diversity in our cohort is comparable to other cohorts.

\section{ASSOCIATION OF KIR AND MHC CLASS I POLYMORPHISMS WITH PROGRESSION TO AIDS}

We initially tested whether the presence of individual KIR genes is correlated, for example due to close genetic linkage, which has hitherto not been determined for rhesus macaque KIR genes. Of particular interest for subsequent genetic association analyses with SIV infection outcome are strong correlations as well as distinctly different correlations in HVL compared to LVL. The latter may occur in the vicinity of functionally relevant genes when specific haplotypes associate with HVL outcome. As can be seen in Table 2, correlation between KIR3DL05-KIR3DSO5KIR3DL10 and KIR3DSW08-KIR3DLW03-KIR3DSW09 strongly suggests that these are neighboring genes (in the given order). Stronger correlation in HVL compared to LVL animals may indicate some functional relevance of these gene sets to infection outcome and differences in KIR haplotypes. 

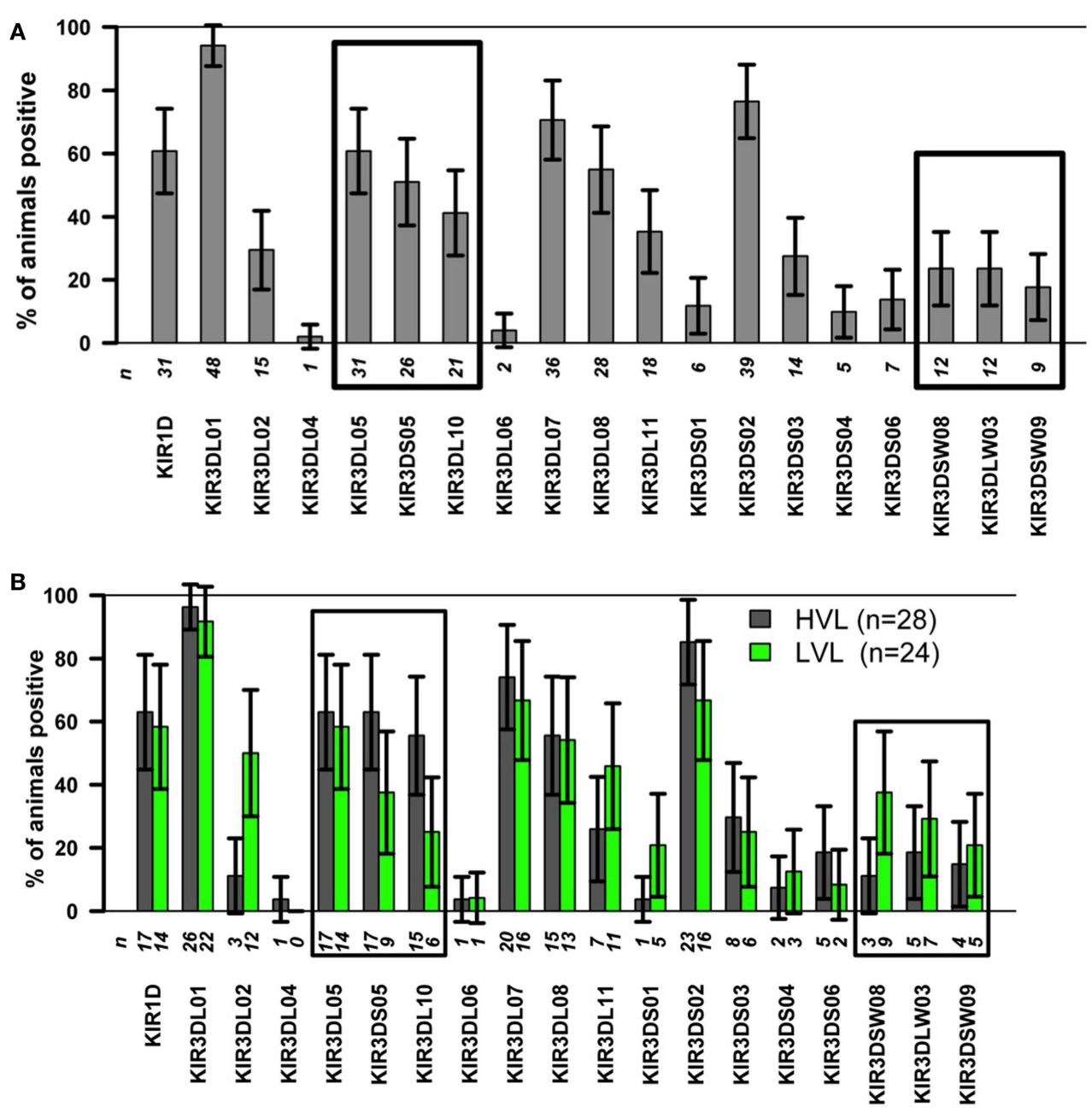

c KIR3DL02 or KIR3DL05 positive animals

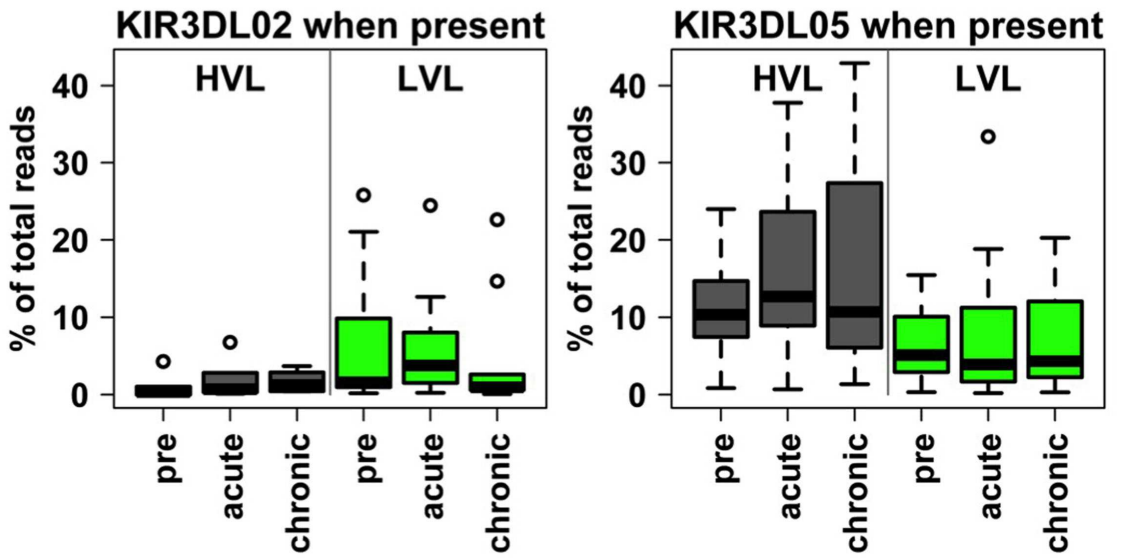

FIGURE 4 | $K I R$ genes in SIV infected rhesus macaques with slow ( $L V L, n=24$ ) and rapid (HVL, $n=28$ ) progression to AIDS. (A,B) Relative percentage of animals displaying the KIR transcript (bar height) with $95 \%$ confidence limits (error bars). The number $n$ of animals transcribing the respective $K I R$ gene is given below the bars. (A) Complete cohort of $n=52$ rhesus macaques. Boxes indicate correlated KIR genes (KIR3DL10-KIR3DSO5-KIR3DL05; KIR3DSW08-KIR3DLW03-KIR3DSW09; see Table 2). (B) Cohort stratified into HVL $(n=28)$ and $\operatorname{LVL}(n=24)$ animals. (C) KIR transcript frequencies of animals transcribing the KIR3DLO2 or the KIR3DLO5 gene before (pre) and during different time points after infection (acute, chronic). 
Table 2 | Correlation of KIR transcript presence at pre-infection.

\begin{tabular}{|c|c|c|c|c|c|c|}
\hline & \multicolumn{2}{|c|}{ Whole cohort $(n=52)$} & \multicolumn{2}{|c|}{ HVL animals $(n=28)$} & \multicolumn{2}{|c|}{ LVL animals $(n=24)$} \\
\hline & KIR3DSO5 & KIR3DLW03 & KIR3DS05 & KIR3DLW03 & KIR3DS05 & KIR3DLW03 \\
\hline$K I R 3 D L 10^{a}$ & $0.74\left(p=5.2 \times 10^{-10}\right)$ & & $0.70\left(p=4.3 \times 10^{-5}\right)$ & & $0.75\left(p=2.9 \times 10^{-5}\right)$ & \\
\hline KIR3DLO5 & $0.42(p=0.0023)$ & & $0.84\left(p=3.9 \times 10^{-8}\right)$ & & $-0.04(p=0.84)$ & \\
\hline KIR3DSW08 ${ }^{\mathrm{b}}$ & & $0.56\left(p=1.6 \times 10^{-5}\right)$ & & $0.74\left(p=9.6 \times 10^{-6}\right)$ & & $0.45(p=0.027)$ \\
\hline KIR3DSW09b & & $0.35(p=0.012)$ & & $0.61\left(p=8.0 \times 10^{-4}\right)$ & & $0.12(p=0.57)$ \\
\hline
\end{tabular}

Displayed correlations (Pearson estimate, $p$ value) were strong enough to have $p \leq 10^{-3}$ in HVL or LVL (significances in bold, Bonferroni: $p \leq 0.05 / 240=2.08 \times 10^{-4}$ for testing pair wise correlations of 16 KIRs in HVL and LVL). KIR3DL04 (2\%), KIR3DLO6 (4\%), KIR3DL01 (92\%) were not tested.

${ }^{a}$ KIR3DL10 tended to correlate with KIR3DLO5 in the whole cohort ( $r=0.35, p=0.013$ ) and in HVL ( $\left.r=0.55, p=0.003\right)$ but not in LVL ( $\left.r=0.10, p=0.65\right)$.

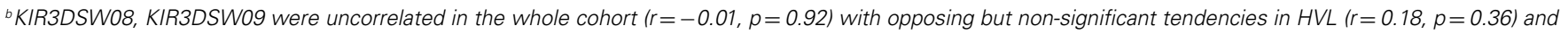
$L V L(r=-0.19, p=0.39)$

For KIR association analyses (Table 3) we converted levels of $K I R$ transcript at pre-infection into binary variables that were subsequently used as covariate(s). Target traits for association analyses included HVL outcome (logistic regression), survival time (Cox regression), NK cell number and functional data (linear regressions). KIR effect estimates were mutually adjusted in a single multi-KIR model of HVL outcome, which was the main target (Table 3, first column, significances $p \leq 0.05$ in bold; excluded were KIRs with small strata size, and KIR3DSO5 and KIR3DLW03 because of strong correlations with neighboring $K I R$ genes as shown in Table 2). All other association analyses in Table 3 employed single-KIR models. Furthermore, the frequency of $K I R$ transcript sequence reads at pre- and acute-infection (where NK cell numbers were high and comparable across HVL and LVL) was jointly analyzed in KIR-positive animals as a longitudinal metric target for association with HVL outcome (Table 3, last column).

The presence of transcripts for KIR3DL02 and KIR3DSW08 was associated significantly with LVL outcome (see Table 3: significant odds ratios $=1$ and Figure 4B). The observable weaker power of the KIR3DSW08 gene is attributed to its low frequency. Additionally, a trend for the even rarer gene KIR3DS01 to be protective was identified by the single-KIR model (Table 3, OR $0.15, p=0.0504)$. KIR3DSO2 was detected as a risk gene for HVL outcome, especially when KIR correlations were accounted for in the multi-KIR model (Table 3; OR 46.5, $p=0.008$ ). Furthermore, KIR3DL10 associated with HVL outcome (Table 3, OR $3.75, p=0.025)$ in single-KIR analysis; with a similar trend for correlated gene KIR3DS05 (Table 3; OR 2.83, $p=0.068$ ), whereas the multi-KIR model implicated presence of KIR3DL05 to associate with LVL outcome (Table 3, OR 0.09, $p=0.037$ ). This difference arises from the intricate correlation structure between KIR3DL05-KIR3DS05-KIR3DL10 described above. The association of KIR3DS05 and KIR3DL10 with HVL outcome was underscored by subsequent epistasis analyses with $M H C$ class $I$ alleles (see below).

Table 3 further illustrates that the association of $K I R$ genes with HVL outcome is mirrored in clinical (survival time) and functional (decline of blood NK cell numbers, sustained blood NK cell proliferation, and loss of blood NK cell lytic function) parameters. Indeed, transcription of the protective KIR genes 3DLO2 and
$3 D S W 08$ is in both cases significantly associated with (1) longer survival time (3DL02: HR 0.24, $p=0.005$; 3DSW08: HR 0.29, $p=0.020),(2)$ higher numbers of blood NK cells (3DL02: +220.7 , $p=0.006$; 3DSW08: $+222.8, p=0.014$ ), and (3) lower numbers of proliferating NK cells (3DL02: $-15.7 \%, p=0.005$; 3DSW08: $-13.2 \%, p=0.025)$. Expression of KIR3DLO2 is further associated with better preservation of lytic capability $(+6.7 \%$ CD $107 \mathrm{a}$ positive, $p=0.033$ ) and higher transcript frequencies in LVL animals compared to HVL animals (Table 3; Figure 4C, $p=0.033$ ). Expression of the putatively protective KIR3DS01 gene is associated with longer survival time (HR $0.20, p=0.041)$ and higher NK cell numbers $(+243.9, p=0.042)$ and by trend also with less NK cell proliferation $(-15.5 \%, p=0.076)$ (Table 3), further underscoring the finding that KIR3DSO1 might be a protective $K I R$ gene. Along the same line, transcription of the risk genes KIR3DL10 and KIR3DSO2 is in both cases associated with shorter survival times (KIR3S02: HR 2.96, $p=0.043$ : KIR3DL10: borderline significance HR 1.96, $p=0.088)$, and with a decrease in NK cell numbers (KIR3DL10: $-180.5, p=0.017$; KIR3DS02: borderline significance $-169.0, p=0.065)$. KIR3DL10 is significantly associated with reduced lytic activity of NK cells $(-6.4 \%$ CD 107apositive cells, $p=0.035$ ) and both KIR3DL10 and KIR3DS02 show a trend for sustained proliferating NK cells at 8-12 weeks p.i. (both $p=0.056$ ) (Table 3 ). Of note, all single-KIR effect estimates in Table 3 for KIR3DSO2 are remarkably similar to those of correlated KIR genes 3DL10, 3DS05 across all analyses. Moreover, the most frequent KIR gene KIR3DL05 in the correlated triplet (3DL05-3DSO5-3DL10) and the highly frequent KIR3DSO2 tend to correlate (whole cohort: $r=0.41, p=0.0031$, HVL stratum: $r=0.54, p=0.0034$, LVL stratum: $r=0.30, p=0.156$, not significant after Bonferroni correction). Notably, KIR3DL05 is associated with higher transcript frequencies in HVL animals compared to LVL animals (Table 3; Figure 4C, $p=0.022$ ).

Furthermore, we determined the transcribed $M H C$ class $I$ alleles and identified 68 distinct $M a m u-A$ and $M a m u-B$ transcripts in the entire cohort of 52 rhesus macaques (see Table S2 for individual data and Table S3 for MHC class I gene/allele frequencies in Supplementary Material), with an average of 10 distinct transcripts per individual. We frequently observed coincidence of particular MHC class I transcripts (see Table S4 in Supplementary Material) 
Table 3 | KIR association with HVL outcome, survival and NK cell function

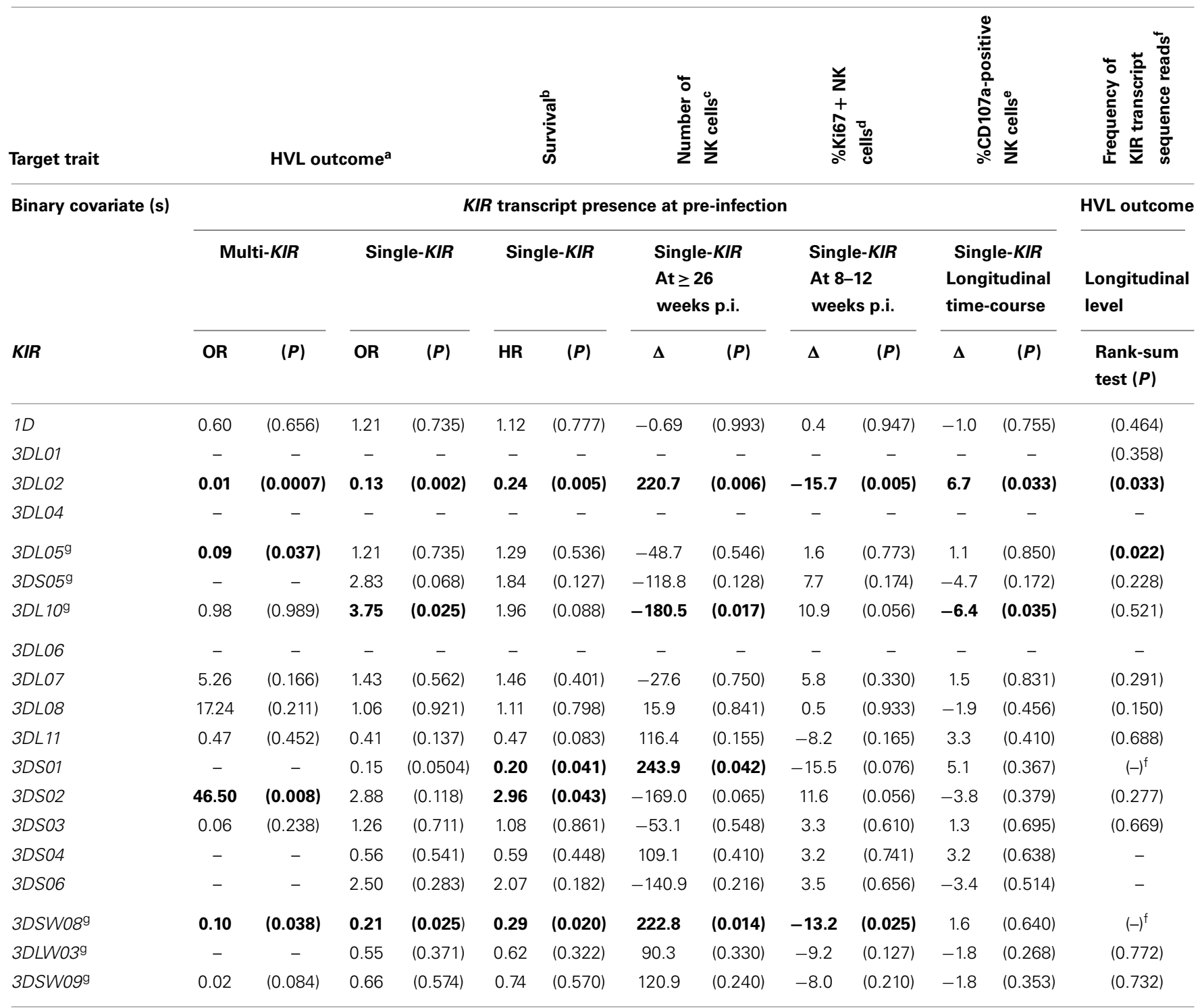

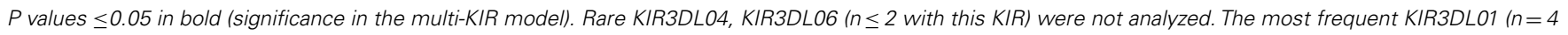
without this KIR) was only analyzed for frequency of KIR transcript sequence reads.

${ }^{a}$ Logistic regression for target HVL outcome, with a single KIR or multiple KIR covariate(s): odds ratio (OR, <1 for protective KIR) and p value (P) (likelihood ratio test, $\chi^{2}$ statistic). ORs in the multi-KIR model are mutually adjusted within a single model [for KIRs with strata sizes $\geq 9$ animals; excluding 3DSO5, 3DLW03 due to strong correlations (see Table 2)].

${ }^{b}$ Cox proportional hazards model with a single KIR covariate: hazard ratio (HR, $<1$ for protective KIR) and $p$ value (P) (likelihood ratio test, $\chi^{2}$ statistic).

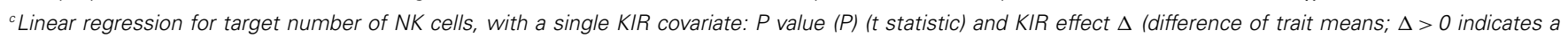
protective KIR that associates with higher NK cell numbers at $\geq 26$ weeks p.i.).

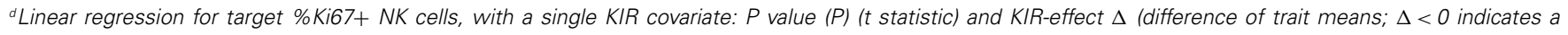
protective KIR that associates with moderated NK cell proliferation at 8-12 weeks p.i.).

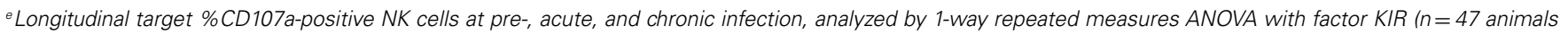

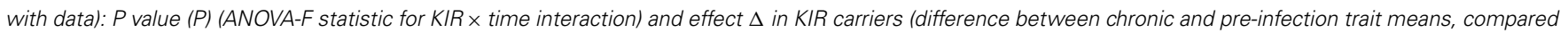

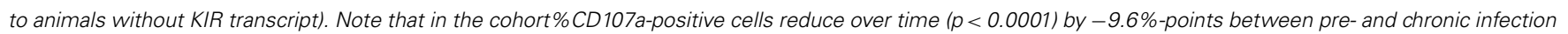
(see Figure 2). Thus, a KIR effect of $0<\Delta<9.6$ implies moderated loss-of-function (protective KIR), $\Delta<0$ enhanced loss-of-function (deleterious KIR).

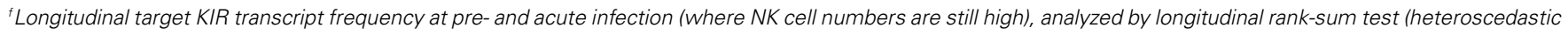

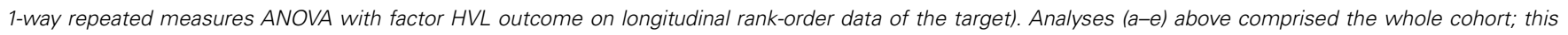
analysis (f) only KIR positive animals [see Figure 4B for subsample sizes; rare KIR's, e.g., KIR3DS01 (5 LVL, 1 HVL) and KIR3DSW08 (9 LVL, 3 HVL) were not analyzed]. $P$ values $(P)$ for association of KIR transcript frequency with HVL outcome; KIR3DL02 and KIR3DLO5 are illustrated in Figure 4C

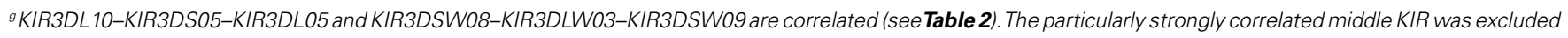
from the multi-KIR model. 
Table 4 | KIR3DS05, KIR3DL10 epistasis with MHC class I alleles on HVL risk

\begin{tabular}{|c|c|c|c|c|c|c|c|c|}
\hline \multirow[b]{3}{*}{$\begin{array}{l}\text { MHC } \\
\text { class I }\end{array}$} & \multicolumn{4}{|c|}{ KIR3DS05 } & \multicolumn{4}{|c|}{$K I R 3 D L 10$} \\
\hline & MHC class I & KIR3DS05 & Epistasis & $P^{a}$ & MHC class I & $K I R 3 D L 10$ & Epistasis & $P^{a}$ \\
\hline & $\begin{array}{c}\mathrm{OR}_{\mathrm{MHC}} \\
\text { ( } p \text { value })\end{array}$ & $\begin{array}{c}\text { OR }_{\mathrm{KIR}} \\
\text { ( } p \text { value })\end{array}$ & $\begin{array}{c}\text { OR }_{\text {MHCxKIR }} \\
\text { (p value) }\end{array}$ & & $\begin{array}{c}\mathrm{OR}_{\mathrm{MHC}} \\
\text { ( } p \text { value) }\end{array}$ & $\begin{array}{c}\text { OR }_{\mathrm{KIR}} \\
\text { ( } p \text { value })\end{array}$ & $\begin{array}{c}\text { OR }_{\text {MHCxKIR }} \\
\text { ( } p \text { value) }\end{array}$ & \\
\hline$A 1 * 001$ & $1.50(p=0.622)$ & $21.60(p=0.009)$ & $0.035(p=0.020)$ & 0.041 & $1.40(p=0.654)$ & $21.60(p=0.009)$ & $0.036(p=0.024)$ & 0.041 \\
\hline$A 1 * 004$ & $11.00(p=0.015)$ & $7.33(p=0.034)$ & $0.20(p=0.224)$ & & $3.64(p=0.101)$ & $3.25(p=0.167)$ & $1.10(p=0.942)$ & \\
\hline$B * 012$ & $0.50(p=0.471)$ & $1.25(p=0.755)$ & $10.00(p=0.087)$ & & $0.40(p=0.320)$ & $1.20(p=0.811)$ & $25.00(p=0.036)$ & \\
\hline$B * 046$ & $2.67(p=0.283)$ & $4.40(p=0.044)$ & $0.26(p=0.272)$ & & $1.30(p=0.745)$ & $3.66(p=0.084)$ & $1.03(p=0.984)$ & \\
\hline$B * 057$ & $6.42(p=0.040)$ & $6.60(p=0.028)$ & $0.17(p=0.153)$ & & $2.60(p=0.221)$ & $4.33(p=0.089)$ & $0.58(p=0.659)$ & \\
\hline$B * 072$ & $10.29(p=0.047)$ & $20.01(p=0.014)$ & $0.054(p=0.043)$ & & $3.00(p=0.179)$ & $8.00(p=0.029)$ & $0.29(p=0.330)$ & \\
\hline
\end{tabular}

Displayed are all model term estimates (main effects and epistasis: odds ratio's OR (p values), $p \leq 0.05$ in bold) for twelve models of KIR-MHC class I polymorphism pairings. The two correlated KIR risk genes KIR3DSO5 and KIR3DL 10 were coded for transcript presence at pre-infection. The MHC class I alleles were coded for transcript presence, did not correlate significantly, and were frequent with sufficient strata sizes. The simultaneous risk estimates $O R_{M H C}, O R_{K I R}$ and OR $R_{M H C X K I R} q_{u a n t i f y}$

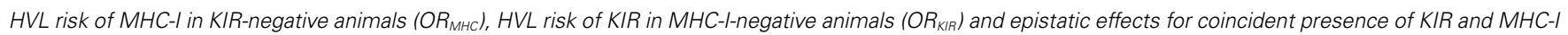
$\left(O R_{M H C x K I R}\right)$. Animals with coincident MHC-I and KIR presence have the HVL risk OR $R_{M H C} \cdot O R_{K I R}$. OR $R_{M H C x K I R}$ compared to animals with coincident MHC-I and KIR absence. a Probability that - under the null-hypothesis of no epistasis and no genetic associations - two or more $p \leq 0.05$ for six epistasis tests of MHC class I alleles with a KIR gene. This probability was estimated based on 10,000 permutations of the HVL/LVL group assignment.

that were previously found to co-occur on distinct MHC class I haplotypes $(29,30)$. It should be noted that rhesus macaque $M H C$ class I haplotypes display extensive CNV of Mamu-B genes (29, 31-33). We found Mamu-A1*004 transcripts more frequently in the HVL than in the LVL stratum (OR 3.7, $p=0.029$ ) (see Table S3 in Supplementary Material), and in accord with previous studies also Mamu-A $1^{\star} 001$ (OR 0.4, $p=0.163$ ) and the rare allele Mamu$B^{\star} 008$ (OR $0.0, p=0.208$ ) more frequently in the LVL than in the HVL stratum (not reaching significance for the latter two).

\section{ANALYSES OF COMBINATIONS OF KIR AND MHC CLASS I POLYMORPHISMS AND PROGRESSION TO AIDS}

KIR3DL1/3DS1 in combination with HLA-Bw4-80I allotypes (in particular HLA-B ${ }^{\star} 57$ ) are associated with slow progression to AIDS in human HIV infection $(8,9,11,34)$. Knowledge of the contribution of similar combinations of KIR and MHC class I alleles is currently not available in the model of SIVmac infection of rhesus macaques.

Sufficient strata sizes for epistasis analyses of KIR and $M H C$ class I transcript presence were available for MHC class I alleles with cohort frequencies between 34.6 and $46.2 \%$ when combined with $K I R$ genes with about $50 \%$ sample frequency. Of these KIR genes, the correlated putative risk genes KIR3DS05, KIR3DL10 were the most interesting. Analyses of epistasis on HVL outcome (Table 4) consistently revealed higher power for KIR3DS05 as well as deleterious effects of KIR3DS05 and Mamu-A1 ${ }^{\star} 004$. Epistasis test $p$ values in Table 4 were not particularly significant. However, observing two (out of six) $p \leq 0.05$ only had a probability $p=0.041$ under the null-hypothesis of no epistasis and no genetic associations. Moreover, model estimates of KIR3DS05 and KIR3DL10 with Mamu-A1 ${ }^{\star} 001$ were remarkably consistent. They indicate that the presence of KIR3DS05 or KIR3DL10 transcripts in Mamu-A1 ${ }^{\star} 001$-negative animals associated with a 21.6-times higher HVL risk $(p=0.009)$. However, presence of $M a m u-A 1^{\star} 001$ transcripts annulled this detrimental effect of KIR3DS05, KIR3DL10 (epistasis $p \leq 0.024$; yielding an odds ratio of 1.1 for combined presence of Mamu-A $1^{\star} 001$ with KIR3DS05 or KIR3DL10).

Whereas $M a m u-B^{\star} 012$ per se did not influence disease outcome according to our analysis (Table 4), there was a tendency of a higher HVL risk in animals with coincident presence of Mamu- $B^{\ngtr} 012$ with KIR3DS05 or KIR3DL10 transcripts (potential epistasis; note also the consistency of model estimates of Mamu- $B^{\ngtr} 012$ with KIR3DS05 and KIR3DL10). Furthermore, it is of interest to note that Mamu-A1 ${ }^{\star} 004, M a m u-B^{\star} 012$, and Mamu$B^{\star} 057$ co-occur on $M H C$ class $I$ haplotypes that were previously reported for other rhesus macaques of Indian origin with rapid or intermediate SIV-disease progression (30).

While ORs had considerable estimation uncertainty (as is common in epistasis analyses), the epistasis of Mamu-A1 ${ }^{\star} 001$ and Mamu- $B^{\star} 012$ with KIR3DS05, and KIR3DL10 on HVL outcome was also observed for viral copy numbers in GIT (Figure 5), and for survival time (Figure 6). In $M a m u$ - $A 1^{\star} 001$-negative animals, viral copy numbers in GIT were particularly high in animals expressing KIR3DS05 and KIR3DL10: $23.9(p=0.0081)$ or $21.8(p=0.017)$ more copies on average, respectively (Figure 5A). A similar trend can be seen in animals expressing the correlated KIR3DL05 gene $(17.2, p=0.076)$. However, presence of $M a m u-A 1^{\star} 001$ transcripts is associated with an effect that is contrary to the KIR3DL05, KIR3DS05, and KIR3DL10 main effects. In animals expressing KIR3DS05, the presence of $M a m u-A 1^{\ngtr} 001$ transcripts was associated with decreased viral copies by -31.6 on average $(p=0.015)$. Similar trends were seen in animals expressing KIR3DL10 or KIR3DL05, where the presence of Mamu-A $1^{\star} 001$ transcripts coincided with decreased viral copy numbers by $-17.3(p=0.19)$ or $-20.6(p=0.13)$ on average (Figure 5A). Coincident presence of Mamu-B ${ }^{\star} 012$ with KIR3DL05, KIR3DS05 or KIR3DL10 transcripts resulted in $32.8(p=0.0173), 34.7(p=0.0091)$ or 


\section{A Epistasis of KIR3DL05, KIR3DS05, KIR3DL10 with Mamu-A1*001}
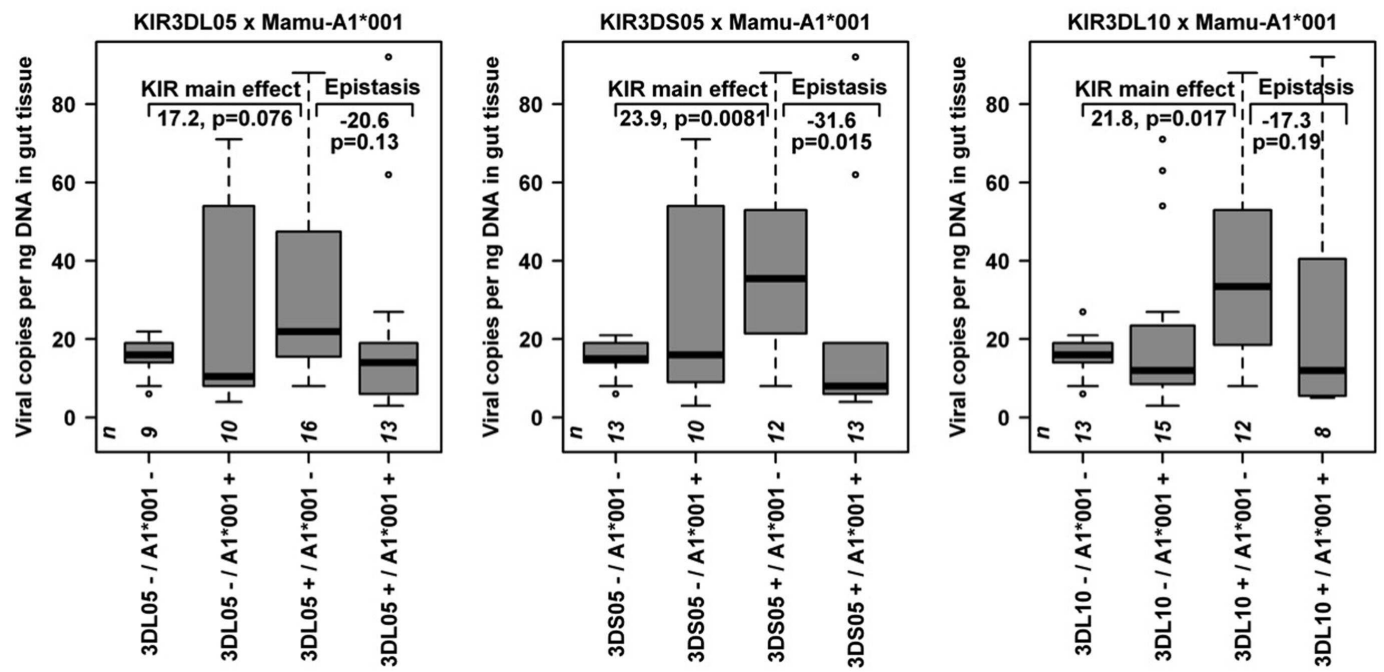

B Epistasis of KIR3DL05, KIR3DS05, KIR3DL10 with Mamu-B*012
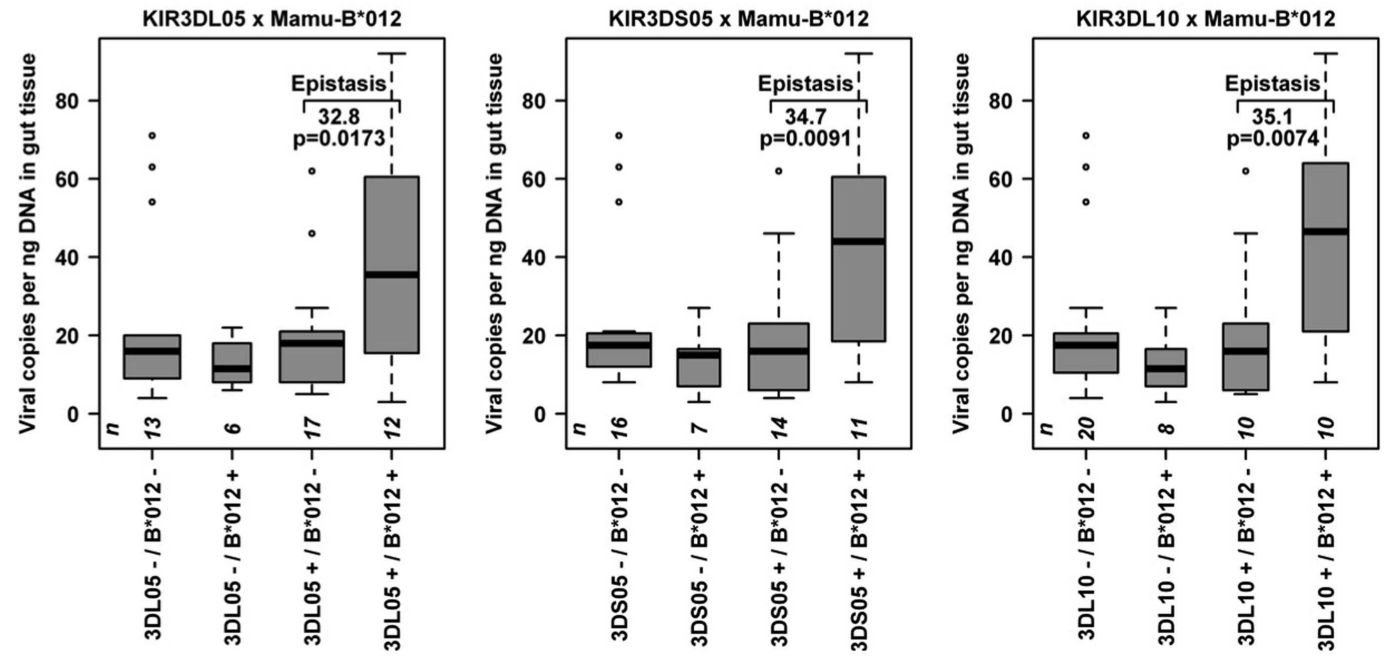

FIGURE 5 | Epistasis of the correlated KIR3DL05-KIR3DS05-KIR3DL 10 genes with $M a m u-A 1 * 001$ and $M a m u-B * 012$ on viral copy numbers in GIT. The boxplots of viral copy numbers stratify for presence $(+)$ and absence (-) of Mamu-A1*001 or Mamu-B012 and the respective KIR (left panels: KIR3DL05, middle panels: KIR3DS05, right panels: KIR3DL 10) transcripts. Sample sizes $n$ are displayed at the bottom of the graphs. (A) Illustrated are the KIR main effects (directly observed in Mamu-A1*001-negative animals) and the epistatic effects of Mamu-A ${ }^{*} 001$ in KIR3DLO5-, KIR3DSO5-, or KIR3DL 10-positive animals. (B) The epistasis model with Mamu- $B^{*} 012$ revealed consistent epistatic effects but no KIR main effects, suggesting that the $K I R$ main effects (see Table $\mathbf{3}$ and this figure, part $\mathbf{A}$ ) may be marginal effects from an epistasis with Mamu- ${ }^{*} 012$. $35.1(p=0.0074)$ more viral copies on average, respectively (Figure 5B), confirming the suspected epistasis. Interestingly, no $K I R$ main effects were seen in the epistasis models with Mamu$B^{\star} 012$ (Figure 5B). This suggests that the KIR3DS05, KIR3DL10 main effects in the single-KIR analyses in Table 3 as well as in Figure 5A may be marginal effects from an epistasis with Мamu- $B^{\star} 012$.

Figure 6 illustrates the fact that the presence of KIR3DL10 transcripts when combined with lack of Mamu-A1*001 or with presence of $M a m u-B^{\star} 012$ transcripts also led to shorter survival times, confirming the epistasis. Highly similar results were obtained for the correlated but the more rare KIR3DS05 gene and for KIR3DL05 (not shown), albeit with weaker power.

\section{DISCUSSION}

Our understanding of the biology of NK cells continues to evolve. Besides its killer function, data supporting the ability of cells within this lineage to become educated, acquire memory and participate in a number of immune regulatory function are accumulating (35-39). Consequently, the role of NK cells in HIV-1 infection 

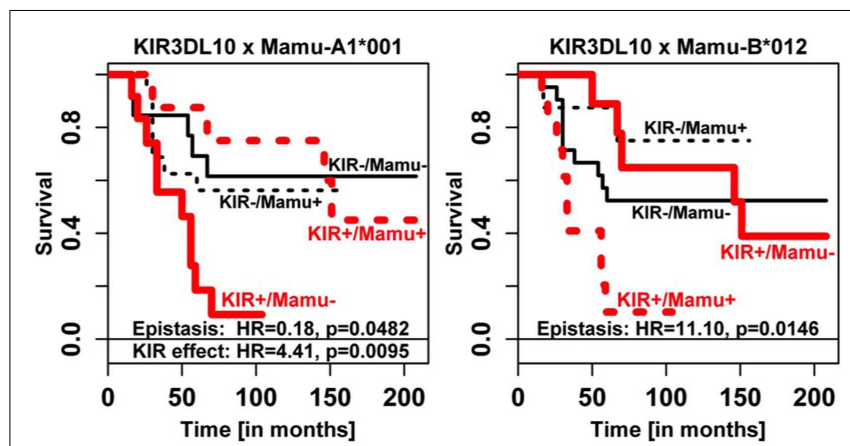

FIGURE 6 | Epistasis of Mamu-A1*001 and Mamu-B*012 with KIR3DL 10 on survival time. The data for KIR3DSO5 are highly similar and are, therefore, not shown. Epistasis effect estimates on survival were consistent for KIR3DL 10 (see figure) and KIR3DS05

(KIR3DS05 × Mamu-A1 *001: detrimental KIR effect HR 4.32, $p=0.0102$, and epistasis HR 0.20, $p=0.0532 ;$ KIR3DSO5 $\times$ Mamu- $B^{*} 012$ : epistasis HR $6.35, p=0.0535)$. The more frequent $K I R 3 D L 10$ had higher statistical power.

and its corresponding non-human primate model has attracted much attention in AIDS research $(20,34,40,41)$. Particular attention in human HIV-1 infection has been paid to genetic factors that modulate NK-cell function and susceptibility to disease. Key determinants of NK-cell activation are the highly polymorphic KIR receptors and their MHC class I ligands. While human KIR3DL1/S1 allotypes in combination with HLA-B allotypes carrying the serological Bw4 epitope with an isoleucine residue at position 80 (HLA-Bw4-80I) were identified as protective, such combinations were hitherto not known in the rhesus macaque SIV infection model.

Based on viral load at set point that correlated well with clinical course (time to the development of AIDS) and absolute levels of peripheral blood CD4+ T cells, we grouped rhesus macaques experimentally infected with SIVmac into "HVL" and "LVL" strata. In contrast to LVL animals that had a relatively benign disease course, we found a progressive loss of peripheral blood NK-cell numbers after peak viraemia in animals rapidly succumbing to SIV-mediated disease (HVL). Further distinguishing HVL from LVL animals is a considerably higher percentage of proliferating $(\% \mathrm{Ki} 67+)$ peripheral blood NK cells in HVL animals as long as 8-12 weeks p.i. (corresponding roughly with viral set point) when NK cell numbers were still high and comparable in both strata. This "proliferating" phenotype of NK cells in HVL animals (Figure 2B), however, is not associated with increased cytotoxic activity as evidenced by decreased CD107a-positive NK cells (Figure 3), indicating dysfunction of NK cells. Dysfunctional NK cells have been reported in HIV-1-infected individuals (4244) and in SIV-infected rhesus macaques (45). Recent studies highlighted an important role of NK cells in the regulation of CD4+ and CD8+ $\mathrm{T}$ cell subsets in various viral infections (38, $46,47)$. For example depending on the viral titer, LCVM infection is lethal to mice, but results in viral clearance if NK cells are depleted (38) or NK-cell deficient mice are used (46). The regulatory role of $\mathrm{NK}$ cells is exerted via killing of CD4+ or CD8+ $\mathrm{T}$ cells, leading to failure of CD8+ T-cell support and CD8+ T-cell responses. Consequently, depletion of NK cells improves
CD8+ T-cell killing of virus-infected cells, enhances T-cell immunity and reduces immune pathology. Depending on the virus, such killing of T cells is perforin dependent or is achieved indirectly via cytokines released by NK cells. The reduced degranulation of NK cells in the HVL animals leads us to hypothesize that the T-cell response is affected in the SIV-infected HVL animals via NK cells' cytokine secretion. Indeed, it has been proposed that cytotoxic CD16 + NK cells have only a limited role in the control of SIV (48). According to our data, this regulation obviously preferentially affects the CD4+ as compared with the CD8+ T-cell subset (Figure 1) and might be particularly effective in HVL animals 412 weeks p.i., a time point when NK cell numbers are still high and about $50 \%$ of these NK cells are proliferating (activated) in contrast to only $10-20 \%$ NK cells in the LVL cohort.

Control of lentiviruses might be influenced by NK cells at the local viral entry site with an important contribution by a NK cell subset that is uniquely present in such mucosal tissues (49). Indeed, rhesus macaques infected intra-rectally or intra-vaginally with SIV $_{\text {mac251 }}$ showed some functional differences of mucosal and blood NK cells, including a higher lytic capacity (\% CD107a expression) of mucosal NK cells (50). However, whether differences in KIR expression are evident and responsible for these phenomena is currently not known.

We hypothesized that the observed functional differences of NK cells and the different disease outcomes are due to KIR and $M H C$ class I polymorphisms. However, comprehensive molecular typing of KIR and MHC class I polymorphisms at the DNA level is extremely complicated in rhesus macaques due to large expansions of both gene families and extensive copy-number variations as well as an absence of detailed genomic information of haplotypes (only single KIR and MHC class I haplotypes are completely sequenced). Therefore, we used published nextgeneration sequencing approaches to determine transcripts of both rhesus macaque gene families $(18,27)$ and analyzed the risk of HVL outcome. Our study identified the inhibitory KIR3DLO2 and the activating KIR3DSW08 (and most probably KIR3DSO1) genes as protective factors in the rhesus macaque SIV infection model (Table 3; Figure 4). The activating KIR3DSO2 gene was detected as a risk gene, but only when $K I R$ correlations were accounted for (multi-KIR model, Table 3). Of note, KIR3DSO2 had a certain correlation with the cluster KIR3DL05-KIR3DSO5KIR3DL10, in particular with KIR3DL05 (whole cohort: $r=0.41$, $p=0.0031$; HVL stratum: $r=0.54, p=0.0034$; LVL stratum: $r=0.30, p=0.156$; non-significant after Bonferroni correction), and was in all single KIR analysis consistent with this KIR gene cluster (Table 3). While no specific ligand has so far been reported for KIR3DL02, a suggested ligand for KIR3DSW08 is Mamu$\mathrm{A} 3^{\star} 13$ (51). In accord with this, the Mamu-A3* 13 allele (formerly designated $M a m u-A^{\star} 13$ ) was reported to be associated with longer survival times of SIV-infected rhesus macaques (52) and is present on $M H C$ haplotypes that were associated with slow, but also intermediate and rapid SIV disease progression (30). Unfortunately, frequencies of KIR3DL02 and KIR3DSW08 in the cohort (see Figure 4B, Table S1 in Supplementary Material) were not high enough to include both in the epistasis analyses. In addition, we had to exclude rare KIR genes (KIR3DL04, KIR3DL06; $n \leq 2, \leq 3.8 \%$ ) or KIR genes with high frequency (KIR3DL01; 
$n=48,92.3 \%)$. Nevertheless, as about half of the animals $(46 \%)$ belong to the LVL group, rare or very frequent genes are not expected to be responsible for the observed differences between LVL and HVL animals. Of note, the relative amounts of KIR transcripts were higher in LVL for the KIR3DL02 gene and higher in HVL animals for the KIR3DL05 gene, respectively (Figure 4C), supporting the aforementioned findings. Although we cannot exclude that these $K I R$ genes show increased transcription, a more likely explanation for this observation appears to be a higher percentage of cells expressing these $K I R$ genes, probably due to clonal expansions ( $\mathrm{T}$ cells) or education (NK cells). As we analyzed transcripts from PBMCs, this might pertain to both KIRexpressing NK and T cell subsets. Furthermore, the association of KIR genes with HVL outcome was mirrored in clinical (survival time) and functional (decline of blood NK cell numbers, sustained NK cell proliferation, and loss of NK cell lytic function) parameters.

How can these associations of KIR genes with SIV disease outcome be explained? In general, involvement of inhibitory KIR expressed on cytolytic cells $(\mathrm{NK}, \mathrm{CD} 8+\mathrm{T}, \gamma \delta \mathrm{T})$ may lower the cytolytic activity of these cells toward infected cells (negative effects), but reduced activity may also result in less immune pathology (positive effect). Similarly, involvement of activating KIR proteins may enhance killing of SIV-infected cells by such cytolytic cells (positive effect), but may also evoke immune pathology (negative effect). Thus, beneficial and detrimental effects can be found for both inhibitory and activating KIR proteins and presence/absence polymorphisms in the individual's genotype certainly influence the outcome. In the case of SIV infection we identified genes associated with a beneficial (KIR3DL02, KIR3DSW08) and others with a detrimental (KIR3DL05, KIR3DL10, KIR3DSO2) outcome of infection.

In (uninfected) rhesus macaques, we previously identified KIR expression on $1-3 \%$ of CD4+ T cells, $4-25 \%$ of $\alpha \beta$ CD8 $+\mathrm{T}$ cells, and $9-58 \%$ of $\gamma \delta \mathrm{T}$ cells (26). The role of KIR expression on T cell subsets is not as elaborated as for NK cells. It was previously shown that expression of inhibitory KIR on tumor-infiltrating human CD8+ T cells was associated with decreased effector functions such as interruption of TCR-mediated signaling and failure to rearrange the actin cytoskeleton (53). KIR expression is particularly seen in memory CD8 $+\mathrm{T}$ cells, but the exact role of KIR proteins in the formation of these cells is not known.

As strong linkage disequilibrium of KIR genes can perturb identification of corresponding risk genes in disease association studies, we analyzed coincidence of KIR gene transcripts and analyzed respective correlations. Indeed, KIR3DL05, KIR3DS05, and KIR3DL10 as well as KIR3DSW08, KIR3DLW03, and KIR3DSW09 correlated, respectively (Table 2 ). These correlations are most likely due to underlying haplotypes. Unfortunately, this assumption could not be tested as only a single rhesus macaque KIR haplotype is completely sequenced (54). Yet, these correlations complicated the analyses for KIR3DL05-KIR3DS05-KIR3DL10 as none of these genes show consistent significant associations in all analyses such as for example KIR3DL02. However, for each of the various parameters tested at least one member of this KIR gene cluster shows significant (or borderline significance) association, indicating that cluster 3DL05-3DS05-3DL10 contains a risk KIR
Table 5 | Known interactions of rhesus macaque KIR and MHC class I ligands.

\begin{tabular}{lll}
\hline KIR & Interacting Mamu class I & Reference \\
\hline KIR3DL01 & Mamu-B with Bw4 motif & $(58)$ \\
KIR3DLW03 & Mamu-A1 alleles *001, ${ }^{*} 008 ;{ }^{*} 011$ & $(51)$ \\
KIR3DL05 & Mamu-A1 alleles ${ }^{*} 001,{ }^{*} 002 ;$ Mamu-A3*13 & $(51,57)$ \\
KIR3DL11 & Mamu-A1*008 & $(51)$ \\
KIR3DS05 & Mamu-A1 alleles *001, ${ }^{*} 011$ & $(51)$ \\
\hline
\end{tabular}

gene. Support for this assumption comes from epistasis analyses of KIR with MHC class I alleles. KIR3DS05 and KIR3DL10 only increased the risk of HVL outcome when $M a m u-A 1^{\star} 001$ was absent (odds ratio 21.6), but not when $M a m u-A 1^{\star} 001$ was present (odds ratio 1.1). This finding is particularly interesting as Mamu$\mathrm{A} 1{ }^{\star} 001$ is a well-known protective $\mathrm{MHC}$ class I allotype $(55,56)$ and a ligand of KIR3DL05 $(51,57)$ and KIR3DS05 (51). A summary of the known KIR and MHC class I interactions is shown in Table 5. As Mamu- $A 1^{\star} 001$ is a well-known protective allele, the observed protective effect in epistasis analysis might also be attributed to this MHC class I allele only and not to epistasis with KIR genes. However, this appears rather unlikely given that (1) $35.7 \%$ $(10 / 28)$ of the HVL animals indeed express Mamu-A1*001 (Table S2 in Supplementary Material) and are not protected, and (2) the mere presence of this $M H C$ class I allele does not protect from high gut viral load (Figure 5A, compare for example 3DS05-/A1*001with $\left.3 D S 05-/ A 1^{\star} 001+\right)$. Furthermore, statistical epistasis tests do not become significant because of consistent variant (KIR and $M H C$ ) effects but rather when such variant effects are statistically not independent (i.e., not additive for gut viral load). Thus, Mamu$A 1^{\star} 001$ does not have a consistent protective effect across KIR strata and its effect obviously depends on epistasis with KIR genes. Further, the combined presence of $M a m u-B^{\star} 012$ and KIR3DS05 (OR 10.0, $p=0.087$ ) or KIR3DL10 (OR 25.0, $p=0.036$ ) suggests a higher risk of HVL outcome. The biological significance of such epistasis is indicated by significant differences in gastro-intestinal viral load (Figure 5B) and in significantly shorter survival times of KIR3DL10-positive animals that lack Mamu- $A 1^{\star} 001$ or express Mamu- $B^{\star} 012$ (Figure 6). Furthermore, epistasis analyses of HVL outcome, GIT viral load, and survival times suggest that KIR3DS05 and KIR3DL10 may not be detrimental per se for disease outcome, but only in combined presence with $M a m u-B^{\star} 012$. In accord with our finding of $M a m u-B^{\star} 012$ being a risk factor, this allele was particularly found on several haplotypes that were associated with rapid progression to AIDS (30). Some of the limitations of our analyses that are outlined above might be overcome in future SIV infection studies involving more animals and when complete KIR haplotypes are sequenced.

In a previous report Hellmann and colleagues (20) used Mamu$A 1^{\star} 001$-negative SIV-infected rhesus macaques and found that variations in copy numbers of activating KIR genes associated with control of SIV replication. Yet, the individual gene(s) underlying this control were not further specified. In accord with the data of Hellmann and colleagues, we identified the activating KIR3DSW08 gene (and most likely KIR3DSO1 as well) as protective in our cohort. The identification of the activating KIR3DS05 as a risk 
factor in combination with $\mathrm{Mamu}-B^{\star} 12$ underscores the necessity to study associations at the level of individual genes as well as epistasis of the involved genes.

We conclude that a comprehensive survey of genetic factors that influence SIV-infection outcome needs both MHC class I and KIR genes in addition to other known anti-viral factors such as TRIM5. Such detailed knowledge is important when analyzing respective data of SIV-infected rhesus macaques to exclude any potential bias introduced by these disease-relevant genetic factors.

\section{ACKNOWLEDGMENTS}

The authors are grateful for expert technical assistance of Nico Westphal and Ann E. Mayne and thank Dr. Libby Guethlein (Stanford University, Stanford, USA) for providing the rhesus macaque KIR gene database.

\section{SUPPLEMENTARY MATERIAL}

The Supplementary Material for this article can be found online at http://www.frontiersin.org/journal/10.3389/fimmu.2014.00600/ abstract

\section{REFERENCES}

1. Lanier LL. Evolutionary struggles between NK cells and viruses. Nat Rev Immunol (2008) 8(4):259-68. doi:10.1038/nri2276

2. Chang JJ, Altfeld M. Innate immune activation in primary HIV-1 infection. J Infect Dis (2010) 202(Suppl 2):S297-301. doi:10.1086/655657

3. Bashirova AA, Thomas R, Carrington M. HLA/KIR restraint of HIV: surviving the fittest. Annu Rev Immunol (2011) 29:295-317. doi:10.1146/annurevimmunol-031210-101332

4. Bostik P, Takahashi Y, Mayne AE, Ansari AA. Innate immune natural killer cells and their role in HIV and SIV infection. HIV Ther (2010) 4:483-504. doi:10.2217/HIV.10.28

5. Parham P, Abi-Rached L, Matevosyan L, Moesta AK, Norman PJ, Older Aguilar AM, et al. Primate-specific regulation of natural killer cells. J Med Primatol (2010) 39:194-212. doi:10.1111/j.1600-0684.2010.00432.x

6. Walter L. Major histocompatibility complex class-I-interacting natural killer cell receptors of nonhuman primates. J Innate Immunity (2011) 3:236-41. doi:10.1159/000323932

7. Martin MP, Carrington M. Immunogenetics of HIV disease. Immunol Rev (2013) 254:245-64. doi:10.1111/imr.12071

8. Martin MP, Gao X, Lee J-H, Nelson GW, Detels R, Goedert JJ, et al. Epistatic interaction between KIR3DS1 and HLA-B delays the progression to AIDS. Nat Genet (2002) 31:429-34. doi:10.1038/ng934

9. Song R, Lisovsky I, Lebouché B, Routy J-P, Bruneau J, Bernard NF. HIV Protective KIR3DL1/S1-HLA-B genotypes influence NK cell-mediated inhibition of HIV replication in autologous CD4 targets. PLoS Pathog (2014) 10:e1003867. doi:10.1371/journal.ppat.1003867

10. Yawata M, Yawata N, Draghi M, Little A-M, Partheniou F, Parham P. Roles for HLA and KIR polymorphisms in natural killer cell repertoire selection and modulation of effector function. J Exp Med (2006) 203:633-45. doi:10.1084/ jem.20051884

11. Martin MP, Qi Y, Gao X, Yamada E, Martin JN, Pereyra F, et al. Innate partnership of HLA-B and KIR3DL1 subtypes against HIV-1. Nat Genet (2007) 39:733-40. doi:10.1038/ng2035

12. Alter G, Martin MP, Teigen N, Carr WH, Suscovich TJ, Schneidewind A, et al. Differential natural killer cell-mediated inhibition of HIV-1 replication based on distinct KIR/HLA subtypes. J Exp Med (2007) 204:3027-36. doi:10.1084/jem.20070695

13. Alter G, Rihn S, Walter K, Nolting A, Martin M, Rosenberg ES, et al. HLA class I subtype-dependent expansion of KIR3DS1+ and KIR3DL1+ NK cells during acute human immunodeficiency virus type 1 infection. J Virol (2009) 83:6798-805. doi:10.1128/JVI.00256-09

14. Alter G, Heckerman D, Schneidewind A, Fadda L, Kadie CM, Carlson JM, et al. HIV-1 adaptation to NK-cell-mediated immune pressure. Nature (2011) 476:96-100. doi:10.1038/nature10237
15. Kruse PH, Rosner C, Walter L. Characterization of rhesus macaque KIR genotypes and haplotypes. Immunogenetics (2010) 62:281-93. doi:10.1007/s00251010-0433-4

16. Blokhuis JH, van der Wiel MK, Doxiadis GGM, Bontrop RE. The mosaic of KIR haplotypes in rhesus macaques. Immunogenetics (2010) 62:295-306. doi:10.1007/s00251-010-0434-3

17. Blokhuis JH, van der Wiel MK, Doxiadis GGM, Bontrop RE. The extreme plasticity of killer cell Ig-like receptor (KIR) haplotypes differentiates rhesus macaques from humans. Eur J Immunol (2011) 41:2719-28. doi:10.1002/eji.201141621

18. Moreland AJ, Guethlein LA, Reeves RK, Broman KW, Johnson RP, Parham P, et al. Characterization of killer immunoglobulin-like receptor genetics and comprehensive genotyping by pyrosequencing in rhesus macaques. BMC Genomics (2011) 12:295. doi:10.1186/1471-2164-12-295

19. Bostik P, Kobkitjaroen J, Tang W, Villinger F, Pereira LE, Little DM, et al. Decreased NK cell frequency and function is associated with increased risk of KIR3DL allele polymorphism in simian immunodeficiency virus-infected rhesus macaques with high viral loads. JImmunol (2009) 182:3638-49. doi:10.4049/ jimmunol.0803580

20. Hellmann I, Lim S-Y, Gelman RS, Letvin NL. Association of activating KIR copy number variation of NK cells with containment of SIV replication in rhesus monkeys. PLoS Pathog (2011) 7:e1002436. doi:10.1371/journal.ppat.1002436

21. Weatherall D. The Use of Non-human Primates in Research (2006), pp. 1-153.

22. Mori K, Yasutomi Y, Sawada S, Villinger F, Sugama K, Rosenwith B, et al. Suppression of acute viremia by short-term postexposure prophylaxis of simian/human immunodeficiency virus SHIV-RT-infected monkeys with a novel reverse transcriptase inhibitor (GW420867) allows for development of potent antiviral immune responses resulting in efficient containment of infection. J Virol (2000) 74:5747-53. doi:10.1128/JVI.74.13.5747-5753.2000

23. Villinger F, Brice GT, Mayne AE, Bostik P, Mori K, June CH, et al. Adoptive transfer of simian immunodeficiency virus (SIV) naïve autologous CD4(+) cells to macaques chronically infected with SIV is sufficient to induce long-term nonprogressor status. Blood (2002) 99:590-9. doi:10.1182/blood.V99.2.590

24. Pereira LE, Johnson RP, Ansari AA. Sooty mangabeys and rhesus macaques exhibit significant divergent natural killer cell responses during both acute and chronic phases of SIV infection. Cell Immunol (2008) 254:10-9. doi:10.1016/j. cellimm.2008.06.006

25. Webster RL, Johnson RP. Delineation of multiple subpopulations of natural killer cells in rhesus macaques. Immunology (2005) 115:206-14. doi:10.1111/j. 1365-2567.2005.02147.x

26. Hermes M, Albrecht C, Schrod A, Brameier M, Walter L. Expression patterns of killer cell Immunoglobulin-like receptors (KIR) of NK-cell and T-cell subsets in old world monkeys. PLoS One (2013) 8:e64936. doi:10.1371/journal.pone. 0064936

27. O'Leary CE, Wiseman RW, Karl JA, Bimber BN, Lank SM, Tuscher JJ, et al. Identification of novel MHC class I sequences in pig-tailed macaques by amplicon pyrosequencing and full-length cDNA cloning and sequencing. Immunogenetics (2009) 61:689-701. doi:10.1007/s00251-009-0397-4

28. Malzahn D, Schillert A, Müller M, Bickeböller H. The longitudinal nonparametric test as a new tool to explore gene-gene and gene-time effects in cohorts. Genet Epidemiol (2010) 34:469-78. doi:10.1002/gepi.20500

29. Doxiadis GGM, de Groot N, Otting N, de Vos-Rouweler AJM, Bolijn MJ, Heijmans CMC, et al. Haplotype diversity generated by ancient recombinationlike events in the MHC of Indian rhesus macaques. Immunogenetics (2013) 65:569-84. doi:10.1007/s00251-013-0707-8

30. Sauermann U, Siddiqui R, Suh Y-S, Platzer M, Leuchte N, Meyer H, et al. Mhc class I haplotypes associated with survival time in simian immunodeficiency virus (SIV)-infected rhesus macaques. Genes Immun (2008) 9:69-80. doi:10.1038/sj.gene.6364448

31. Otting N, Heijmans CMC, Noort RC, de Groot NG, Doxiadis GGM, van Rood JJ, et al. Unparalleled complexity of the MHC class I region in rhesus macaques. Proc Natl Acad Sci U S A (2005) 102:1626-31. doi:10.1073/pnas.0409084102

32. Otting N, Heijmans CMC, van der Wiel M, de Groot NG, Doxiadis GGM, Bontrop RE. A snapshot of the Mamu-B genes and their allelic repertoire in rhesus macaques of Chinese origin. Immunogenetics (2008) 60:507-14. doi:10.1007/s00251-008-0311-5

33. Wiseman RW, Karl JA, Bimber BN, O’Leary CE, Lank SM, Tuscher JJ, et al. Major histocompatibility complex genotyping with massively parallel pyrosequencing. Nat Med (2009) 15(11):1322-6. doi:10.1038/nm.2038 
34. Carrington M, Alter G. Innate immune control of HIV. Cold Spring Harb Perspect Med (2012) 2:a007070. doi:10.1101/cshperspect.a007070

35. Sun JC, Beilke JN, Lanier LL. Adaptive immune features of natural killer cells. Nature (2009) 457(7229):557-61. doi:10.1038/nature07665

36. Paust S. Andrian von UH. Natural killer cell memory. Nat Immunol (2011) 131:500-8. doi:10.1038/ni.2032

37. Narni-Mancinelli E, Jaeger BN, Bernat C, Fenis A, Kung S, De Gassart A, et al. Tuning of natural killer cell reactivity by NKp46 and Helios calibrates T cell responses. Science (2012) 335:344-8. doi:10.1126/science.1215621

38. Waggoner SN, Cornberg M, Selin LK, Welsh RM. Natural killer cells act as rheostats modulating antiviral T cells. Nature (2012) 481(7381):394-8. doi:10.1038/nature10624

39. Ebihara T, Jonsson AH, Yokoyama WM. Natural killer cell licensing in mice with inducible expression of MHC class I. Proc Natl Acad Sci U S A (2013) 110:E4232-7. doi:10.1073/pnas.1318255110

40. Jost S, Altfeld M. Control of human viral infections by natural killer cells. Annu Rev Immunol (2013) 31:163-94. doi:10.1146/annurev-immunol-032712100001

41. Hong HS, Rajakumar PA, Billingsley JM, Reeves RK, Johnson RP. No monkey business: why studying NK cells in non-human primates pays off. Front Immunol (2013) 4:32. doi:10.3389/fimmu.2013.00032

42. Naranbhai V, Altfeld M, Karim SSA, Ndung'u T, Karim QA, Carr WH. Changes in natural killer cell activation and function during primary HIV-1 infection. PLoS One (2013) 8:e53251. doi:10.1371/journal.pone.0053251

43. Brunetta E, Hudspeth KL, Mavilio D. Pathologic natural killer cell subset redistribution in HIV-1 infection: new insights in pathophysiology and clinical outcomes. J Leukoc Biol (2010) 88(6):1119-30. doi:10.1189/jlb.0410225

44. Brunetta E, Fogli M, Varchetta S, Bozzo L, Hudspeth KL, Marcenaro E, et al. The decreased expression of Siglec-7 represents an early marker of dysfunctional natural killer-cell subsets associated with high levels of HIV-1 viremia. Blood (2009) 114:3822-30. doi:10.1182/blood-2009-06-226332

45. LaBonte ML, McKay PF, Letvin NL. Evidence of NK cell dysfunction in SIVinfected rhesus monkeys: impairment of cytokine secretion and NKG2C/C2 expression. Eur J Immunol (2006) 36:2424-33. doi:10.1002/eji.200635901

46. Lang PA, Lang KS, Xu HC, Grusdat M, Parish IA, Recher M, et al. Natural killer cell activation enhances immune pathology and promotes chronic infection by limiting CD8+ T-cell immunity. Proc Natl Acad Sci U S A (2012) 109(4):1210-5. doi:10.1073/pnas.1118834109

47. Welsh RM, Waggoner SN. NK cells controlling virus-specific T cells: rheostats for acute vs. persistent infections. Virology (2013) 435:37-45. doi:10.1016/j.virol. 2012.10.005

48. Choi EI, Reimann KA, Letvin NL. In vivo natural killer cell depletion during primary simian immunodeficiency virus infection in rhesus monkeys. J Virol (2008) 82:6758-61. doi:10.1128/JVI.02277-07

49. Reeves RK, Rajakumar PA, Evans TI, Connole M, Gillis J, Wong FE, et al. Gut inflammation and indoleamine deoxygenase inhibit IL-17 production and promote cytotoxic potential in NKp44+ mucosal NK cells during SIV infection. Blood (2011) 118:3321-30. doi:10.1182/blood-2011-04-347260

50. Liyanage NPM, Gordon SN, Doster MN, Pegu P, Vaccari M, Shukur N, et al. Antiretroviral therapy partly reverses the systemic and mucosal distribution of NK cell subsets that is altered by SIVmac251 infection of macaques. Virology (2014) 45(0-451):359-68. doi:10.1016/j.virol.2013.12.003
51. Rosner C, Kruse PH, Hermes M, Otto N, Walter L. Rhesus macaque inhibitory and activating KIR3D interact with Mamu-A-encoded ligands. J Immunol (2011) 186:2156-63. doi:10.4049/jimmunol.1002634

52. Mühl T, Krawczak M, Haaft ten P, Hunsmann G, Sauermann U. MHC class I alleles influence set-point viral load and survival time in simian immunodeficiency virus-infected rhesus monkeys. J Immunol (2002) 169:3438-46. doi:10.4049/jimmunol.169.6.3438

53. Guerra N, Michel F, Gati A, Gaudin C, Mishal Z, Escudier B, et al. Engagement of the inhibitory receptor CD158a interrupts TCR signaling, preventing dynamic membrane reorganization in CTL/tumor cell interaction. Blood (2002) 100:2874-81. doi:10.1182/blood-2002-02-0643

54. Sambrook JG, Bashirova A, Palmer S, Sims S, Trowsdale J, Abi-Rached L, et al. Single haplotype analysis demonstrates rapid evolution of the killer immunoglobulin-like receptor (KIR) loci in primates. Genome Res (2005) 15:25-35. doi:10.1101/gr.2381205

55. Zhang Z-Q, Fu T-M, Casimiro DR, Davies M-E, Liang X, Schleif WA, et al. Mamu- $A^{\star} 01$ allele-mediated attenuation of disease progression in simianhuman immunodeficiency virus infection. J Virol (2002) 76:12845-54. doi:10. 1128/JVI.76.24.12845-12854.2002

56. Mothé BR, Weinfurter J, Wang C, Rehrauer W, Wilson N, Allen TM, et al. Expression of the major histocompatibility complex class I molecule Mamu- $\mathrm{A}^{\star} 01$ is associated with control of simian immunodeficiency virus SIVmac239 replication. J Virol (2003) 77:2736-40. doi:10.1128/JVI.77.4.2736-2740.2003

57. Colantonio AD, Bimber BN, Neidermyer WJ, Reeves RK, Alter G, Altfeld M, et al. Polymorphisms modulate peptide-dependent binding to an MHC Class I ligand with a Bw6 Motif. PLoS Pathog (2011) 7:e1001316. doi:10.1371/journal. ppat.1001316

58. Schafer JL, Colantonio AD, Neidermyer WJ, Dudley DM, Connole M, O'Connor $\mathrm{DH}$, et al. KIR3DL01 recognition of Bw4 ligands in the rhesus macaque: maintenance of Bw4 specificity since the divergence of apes and old world monkeys. Immunol (2014) 192:1907-17. doi:10.4049/jimmunol.1302883

Conflict of Interest Statement: The authors declare that the research was conducted in the absence of any commercial or financial relationships that could be construed as a potential conflict of interest.

Received: 03 October 2014; accepted:07 November 2014; published online: 28 November 2014.

Citation: Albrecht C, Malzahn D, Brameier M, Hermes M, Ansari AA and Walter L (2014) Progression to AIDS in SIV-infected rhesus macaques is associated with distinct KIR and MHC class I polymorphisms and NK cell dysfunction. Front. Immunol. 5:600. doi: 10.3389/fimmu.2014.00600

This article was submitted to NK Cell Biology, a section of the journal Frontiers in Immunology.

Copyright (c) 2014 Albrecht, Malzahn, Brameier, Hermes, Ansari and Walter. This is an open-access article distributed under the terms of the Creative Commons Attribution License (CC BY). The use, distribution or reproduction in other forums is permitted, provided the original author(s) or licensor are credited and that the original publication in this journal is cited, in accordance with accepted academic practice. No use, distribution or reproduction is permitted which does not comply with these terms. 